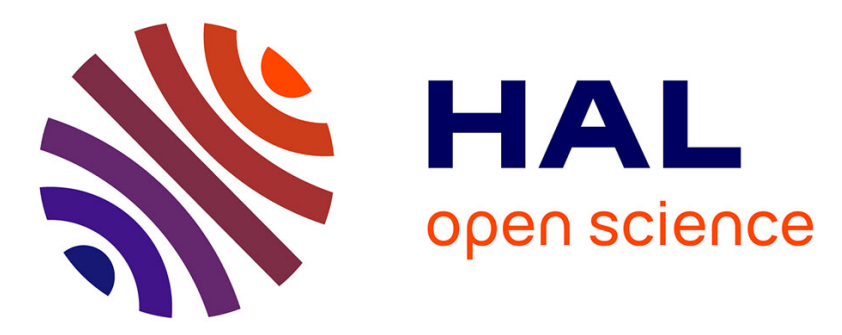

\title{
A fast method for solving microstructural problems defined by digital images: a space Lippmann-Schwinger scheme
}

Julien Yvonnet

\section{- To cite this version: \\ Julien Yvonnet. A fast method for solving microstructural problems defined by digital images: a space Lippmann-Schwinger scheme. International Journal for Numerical Methods in Engineering, 2012, 92 (2), pp.178-205. 10.1002/nme.4334 . hal-00822037}

\section{HAL Id: hal-00822037 \\ https://hal.science/hal-00822037}

Submitted on 7 Sep 2014

HAL is a multi-disciplinary open access archive for the deposit and dissemination of scientific research documents, whether they are published or not. The documents may come from teaching and research institutions in France or abroad, or from public or private research centers.
L'archive ouverte pluridisciplinaire HAL, est destinée au dépôt et à la diffusion de documents scientifiques de niveau recherche, publiés ou non, émanant des établissements d'enseignement et de recherche français ou étrangers, des laboratoires publics ou privés. 


\title{
A fast method for solving microstructural problems defined by digital images : a space-Lippmann-Schwinger scheme
}

\author{
J. Yvonnet ${ }^{1, *}$ \\ 1 Université Paris-Est, Laboratoire Modélisation et Simulation Multi Échelle, UMR 8208 CNRS, \\ 5 Bd Descartes, 77454 Marne-la-Vallée Cedex, France
}

SUMMARY

A fast numerical method is proposed to solve thermomechanical problems over periodic microstructures whose geometries are provided by experimental techniques, like X-ray micro tomography images. In such configuration, the phase properties are defined over regular grids of voxels. To overcome the limitations of calculations on such fine models, an iterative scheme is proposed, avoiding the construction and storage of finite element matrices. Equilibrium equations are written in the form of a Lippmann-Schwinger integral equation, which can be solved iteratively. Unlike previous algorithms based on the Fourier transform, the present scheme strictly operates in the real space domain and removes the numerical Fourier and inverse Fourier transforms at each iteration. For this purpose, the linear operator related to the Lippmann-Schwinger equation is constructed numerically by means of transformation tensors in the real space domain. The convergence and accuracy of the method are evaluated through examples in both steady-state thermics and linear elasticity problems. Computational times are found to scale linearly with the number of degrees of freedom and parallel computations can be carried out straightforwardly. The method is also illustrated on many examples involving complex microstructures, including a problem defined by a micro tomography image.

Copyright (c) 2011 John Wiley \& Sons, Ltd.

KEY WORDS: Complex Microstructures; Lippmann-Schwinger equation; Micro tomography; Computational homogenization; SLS method.

\section{Introduction}

One recent progress in material science is the combination of high resolution imaging techniques and computational methods. Nowadays, realistic three-dimensional geometry of microstructures can be routinely obtained by experimental imagery techniques like X-ray

\footnotetext{
${ }^{*}$ Correspondence to: Julien Yvonnet, Université Paris-Est, 5 Bd Descartes, 77454 Marne-la-Vallée Cedex, France

${ }^{\dagger}$ Please ensure that you use the most up to date class file, available from the NME Home Page at http://www.interscience.wiley.com/jpages/0029-5981/

Contract/grant sponsor: Publishing Arts Research Council; contract/grant number: 98-1846389
} 
microtomography $[20,22]$. After an appropriate image treatment, the obtained data are in the form of three-dimensional grids (voxels) which can be assigned to the properties of each phase. To obtain either the macroscopic thermomechanical properties or the local fields, local equations must be solved by a numerical method. According to the type of experimental devices, the data can contain up to $1024^{3}$ or $2048^{3}$ points rendering the use of classical approaches such as the finite element very costly regarding computational times and memory requirements. On one hand, constructing a mesh matching the interfaces from a voxels grid is a very delicate task. On the other hand, using directly the voxels as elements leads to a huge number of degrees of freedom making the systems resolution highly inefficient.

In $[16,17]$, Moulinec and Suquet proposed an iterative scheme avoiding the construction and decomposition of finite element matrices for periodic microstructures. The idea is to re-write the equilibrium equation in the form of an integral equation which can be solved iteratively. The convolution product and related Green operators are evaluated in the Fourier space. Then an inverse Fourier transform is applied to evaluate the constitutive equations at each iteration in the real space. A similar method has been proposed by Müller [19] to model phase transformations. Improvements of Fast Fourier Transform method can be found in $[11,13,18]$ for dealing with nonlinear problems and arbitrary phase contrasts. See $[2,15,24,27]$ for recent improvement of the convergence in the iterative schemes and [14, 1, 26, 21] for other applications and recent extensions.

In the present work, a similar scheme is proposed, but which avoids the use of the Fourier transform while maintaining the efficiency and convenience of iterative schemes over regular grids. To solve the Lippmann-Schwinger equation in the real space domain, several difficulties arise: (a) the convolution product in the real space domain is a $\mathcal{O}\left(N^{2}\right)$ operation, with $N$ being the number of degrees of freedom of the system; (b) numerical integration of the Green function is a delicate operation, as the operator is highly singular. In the proposed technique, coined as Space Lippmann Schwinger (SLS), these issues are overcome by directly computing the integral operator numerically. This operator is determined in the form of transformation tensors computed by means of the finite element method on a reduced domain. For this purpose, a constant eingenstress is prescribed in a voxel, and the resulting strain field serves to construct the transformation tensors related to the integral operator. The method and resulting algorithms are presented in section 2 for both steady-state thermal and linear elasticity problems. Computational details are provided in section 3. In section 4 several numerical examples are presented to evaluate the convergence, accuracy and efficiency of the method over thermal and elasticity problems. Different examples involving complex microstructures are presented to illustrate the potential of the technique.

\section{Formulation and algorithms}

The aims of the present method are to compute the effective properties of a heterogeneous material or either obtaining a description of local fields in the microstructure. A representative volume element (r.v.e) is assumed known and its physical (e.g. thermal, elastic) properties are defined in a regular grid of voxels (we maintain the word voxel throughout this paper, even though the term pixel would be more appropriate to the present $2 \mathrm{D}$ applications). The r.v.e. is defined in a domain $\Omega \subset \mathbb{R}^{D}, D$ being the space dimension. The phases are assumed perfectly bounded and the microstructure periodic. 


\subsection{Thermal steady-state problem}

For the sake of simplicity, we first provide the ideas of the method in the context of steadystate thermal problems. Given a grid of voxels with assigned thermal conductivity and a macroscopic thermal gradient $\overline{\mathbf{G}}$, the localization problem consists in finding the microscopic gradient $\nabla \mathbf{T}(\mathbf{x})$ and flux fields $\mathbf{q}(\mathbf{x})$ in the microstructure such that

$$
\nabla \cdot(\mathbf{q}(\mathbf{x}))=0 \text { in } \Omega,
$$

where $\nabla \cdot($.$) denotes the divergence operator and where \mathbf{q}(\mathbf{x})$ is related to the gradient through the Fourier's constitutive equation:

$$
\mathbf{q}(\mathbf{x})=-\mathbf{k}(\mathbf{x}) \nabla \mathbf{T}(\mathbf{x}),
$$

$\mathbf{k}$ being a second-order conductivity tensor and $\nabla($.$) the gradient operator. The gradient field$ is such that

$$
\langle\nabla \mathbf{T}(\mathbf{x})\rangle=\overline{\mathbf{G}},
$$

where $\langle$.$\rangle denotes the spatial average over \Omega$. Condition (3) is satisfied for

$$
\nabla \mathbf{T}(\mathbf{x})=\overline{\mathbf{G}}+\nabla \tilde{\mathbf{T}}(\mathbf{x}),
$$

where $\nabla \tilde{\mathbf{T}}(\mathbf{x})$ is a periodic temperature gradient field over $\Omega$. Introducing a reference medium with conductivity $\mathbf{k}^{0}$ and splitting $\mathbf{k}(\mathbf{x})$ into

$$
\mathbf{k}(\mathbf{x})=\mathbf{k}^{0}+\mathbf{k}(\mathbf{x})-\mathbf{k}^{0},
$$

the problem (1) can be written as

$$
\nabla \cdot\left(\mathbf{k}^{0} \nabla \mathbf{T}(\mathbf{x})\right)=-\nabla \cdot\left(\left[\mathbf{k}(\mathbf{x})-\mathbf{k}^{0}\right] \nabla \mathbf{T}(\mathbf{x})\right) .
$$

Setting

$$
\hat{\mathbf{q}}(\mathbf{x})=\left[\mathbf{k}(\mathbf{x})-\mathbf{k}^{0}\right] \nabla \mathbf{T}(\mathbf{x})
$$

Eq.(6) becomes

$$
\nabla \cdot\left(\mathbf{k}^{0} \nabla \mathbf{T}(\mathbf{x})\right)=-\nabla \cdot(\hat{\mathbf{q}}(\nabla \mathbf{T}(\mathbf{x}))) .
$$

Now using (4) we obtain

$$
\nabla \cdot\left(\mathbf{k}^{0} \nabla \tilde{\mathbf{T}}(\mathbf{x})\right)=-\nabla \cdot(\hat{\mathbf{q}}(\nabla \mathbf{T}(\mathbf{x}))) .
$$

As the problem (9) is linear, its solution can be expressed as

$$
\nabla \tilde{T}_{i}(\mathbf{x})=-\Gamma_{i j}^{0} * \hat{q}_{j}(\nabla \mathbf{T}(\mathbf{x})),
$$

or

$$
\nabla \tilde{T}_{i}(\mathbf{x})=-\int_{\Omega} \Gamma_{i j}^{0}(\mathbf{x}-\mathbf{y}) \hat{q}_{j}(\nabla \mathbf{T}(\mathbf{y})) d \Omega
$$


where $\Gamma_{i j}^{0}$ is the periodic Green function related to the linear operator in (9) (see Appendix 7).

Then we have, using (4) and (11):

$$
\nabla T_{i}(\mathbf{x})=\overline{\mathbf{G}}-\int_{\Omega} \Gamma_{i j}^{0}(\mathbf{x}-\mathbf{y}) \hat{q}_{j}(\nabla \mathbf{T}(\mathbf{y})) d \Omega
$$

Eq. (12) is an integral equations, identified as a Fredholm equation of the second type, and also called Lippmann-Schwinger equation (see e.g. [8]). The main interest of such form is that it can be solved iteratively using a development of the linear operator into Neumann series [7]. Writing (12) as

$$
\nabla \mathbf{T}(\mathbf{x})=\mathcal{L}(\nabla \mathbf{T}(\mathbf{x}))+\overline{\mathbf{G}},
$$

we have

$$
\nabla \mathbf{T}(\mathbf{x})=(\mathbb{I}-\mathcal{L})^{-1} \overline{\mathbf{G}} .
$$

The operator $(\mathbb{I}-\mathcal{L})^{-1}$ can be approximated by a Neumann series. If $r(\mathcal{L})<1, r($.$) being the$ spectral radius of the linear operator $\mathcal{L}[7]$ we have

$$
(\mathbb{I}-\mathcal{L})^{-1}(.) \simeq \mathbb{I}+\mathcal{L}(.)+\mathcal{L}(\mathcal{L}(.))+\ldots+\mathcal{L}^{n}(.)
$$

where $\mathbb{I}$ is the identity operator and $\mathcal{L}^{n}($.$) denotes the n-t h$ application of the operator $\mathcal{L}$. From (14) an (15) an iterative scheme (fixed point) can be defined to solve (13) as

$$
\nabla \mathbf{T}^{n+1}(\mathbf{x})=\mathcal{L}\left(\nabla \mathbf{T}^{n}(\mathbf{x})\right)+\overline{\mathbf{G}},
$$

where here $n$ denotes the iteration index. In the present work, we propose a numerical method to solve problems in form of Lippmann-Schwinger equation with an algorithm operating only in the real space domain. Then to apply the iterative scheme (16), a fast method must be provided to evaluate $\mathcal{L}$ efficiently. The Green function $\Gamma^{0}$ can be defined exactly in the real space for many linear partial differential equations (see e.g. [9, 3, 23]). However, its convolution with an arbitrary function given in a discrete form is delicate, as $\Gamma^{0}(\mathbf{x}-\mathbf{y})$ is highly singular at $\mathbf{x}=\mathbf{y}$. Furthermore, the convolution product is a $\mathcal{O}\left(N^{2}\right)$ operation, and its numerical evaluation is prohibitive for large systems. To overcome these difficulty, the proposed Space Lippmann-Schwinger (SLS) method is described as follows. In this framework, the Green's function is not integrated, i.e. the convolution product is not evaluated numerically. Instead, the stress field is assumed constant in each voxel, as the voxel is the elementary unit for image-based simulations. Then the problem of prescribing a unitary eigenstress in one voxel is solved by the finite element method. The resulting solution corresponds to the convolution product of the analytical Green's function with a unitary stress field located in one voxel (square domain in 2D). Using the superposition principle, the complete local strain field is a linear combination of the obtained solutions with respect to actual values of the stress in each voxel. With this procedure, the complicated integration procedure of the Green's function is avoided. We further restrict the presentation to the two-dimensional case, while extension to $3 \mathrm{D}$ is straightforward.

In the following, we will distinguish two different grids; (a) the material grid, related to the r.v.e, containing the piece-wise information of phase properties in a $N_{x} \times N_{y}$ grid and (b) a 


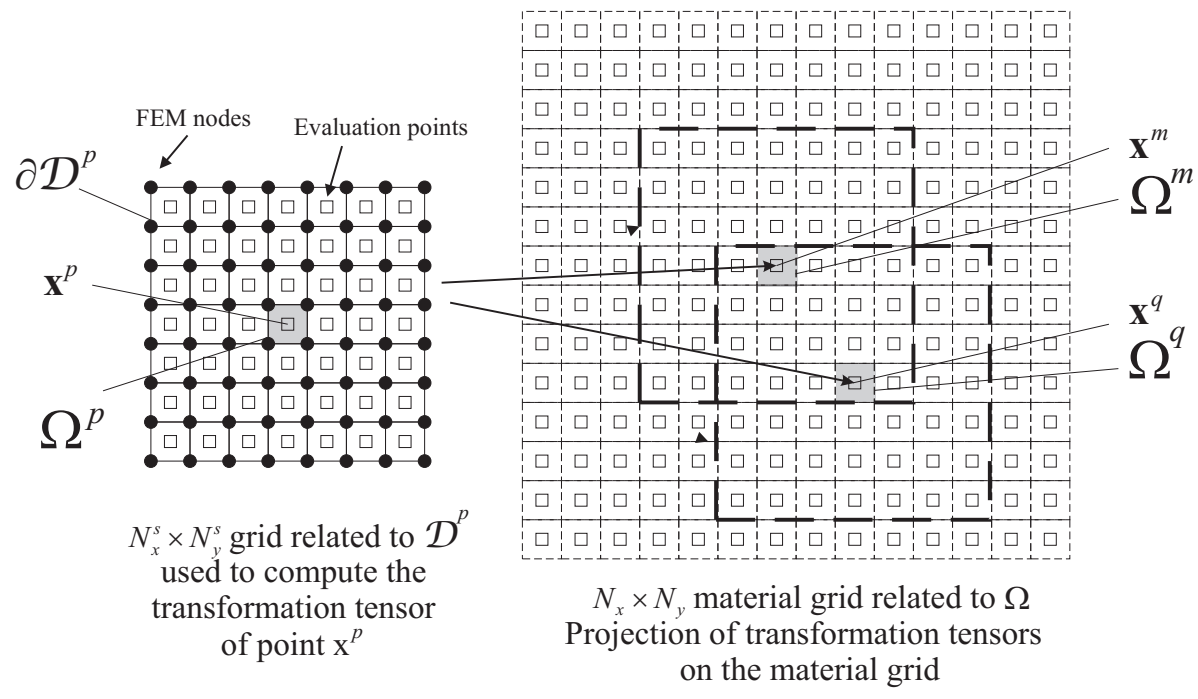

(a)

(b)

Figure 1. (a) Mesh used to compute transformation tensors of a reference point $\mathbf{x}^{p}$; (b) Material grid related to the r.v.e. and projection of the transformation tensors for points $\mathbf{x}^{m}$ and $\mathbf{x}^{q}$.

smaller grid with $N_{x}^{s} \times N_{y}^{s}$ points, utilized to compute the transformation tensors related to the integral operator in the Lippmann-Schwinger equation (see figure 1).

We first assume that the constitutive properties are piece-wise constant within each voxel of the material grid. For the sake of simplicity we also assume here that each phase is isotropic while this is not mandatory. In that context the thermal conductivity $k(\mathbf{x})$ is expressed as:

$$
k(\mathbf{x}) \simeq \sum_{m} \chi^{m}(\mathbf{x}) k^{m} \quad \forall \mathbf{x} \in \Omega,
$$

where $\chi^{m}$ is a characteristic function related to a voxel $m$ in the material grid (see figure 1 (b)), such as $\chi^{m}(\mathbf{x})=1$ for $\mathbf{x} \in \Omega^{m}$ and zero otherwise, $\Omega^{m}$ being a parallelepipedic domain $\Omega^{m}$ centered on the point $\mathbf{x}^{p}$ and $k^{p}$ the conductivity in the voxel $m$.

Invoking the superposition principle we can write

$$
\nabla \tilde{T}_{i}^{n+1}(\mathbf{x})=\sum_{p} \psi_{i j}^{p}(\mathbf{x}) \hat{q}_{j}^{n}\left(\mathbf{x}^{p}\right)
$$

where

$$
\psi_{i j}^{p}(\mathbf{x})=\nabla \phi_{i}^{p j}(\mathbf{x})
$$

are basis functions, or transformation tensors. The variable $\phi^{p j}(\mathbf{x})$ is defined as a temperature field verifying

$$
\nabla \cdot\left(k^{0} \nabla \phi^{p j}(\mathbf{x})\right)=-\nabla \cdot\left(\mathcal{F}^{p j}(\mathbf{x})\right) \text { for } j=1, \ldots, D
$$


with the boundary conditions

$$
\phi^{p j}(\mathbf{x})=0 \text { on } \partial \mathcal{D}^{p}
$$

corresponding to $\nabla \phi^{p j}(\mathbf{x})=\mathbf{0}$ over $\partial \mathcal{D}^{p}$. In (20) $D$ is the problem dimension and $\mathcal{F}^{p j}(\mathbf{x})$ is a prescribed constant flux in $\Omega^{p}$ such that

$$
\mathcal{F}^{p j}(\mathbf{x})=\chi^{p}(\mathbf{x}) \mathbf{e}_{j} .
$$

Eq. (20) can be solved numerically, e.g. by means of the finite element method. Then prescribing the step flux $\mathcal{F}^{p j}(\mathbf{x})$ for each component $j$ independently gives a temperature field solution $\phi^{p j}(\mathbf{x})$ which can be derived to obtain $\nabla \phi_{i}^{p j}(\mathbf{x})$. At this stage two important remarks can be drawn:

1. As $\psi_{i j}^{p}(\mathbf{x})$ are associated to an homogeneous medium in an infinite domain, we have:

$$
\psi_{i j}^{p}(\mathbf{x})=\psi_{i j}^{m}(\mathbf{x}-\mathbf{a}), \quad \mathbf{x}^{m}=\mathbf{x}^{p}-\mathbf{a},
$$

where $\mathbf{a}$ is an arbitrary translation vector. In other words, the temperature solution produced by a step flux in a voxel centered at point $\mathbf{x}^{p}$ is the same that the one produced by a similar flux at a point $\mathbf{x}^{m}$, translated by $\mathbf{a}=\mathbf{x}^{m}-\mathbf{x}^{p}$ (see figure 2 ).

2. As $\psi_{i j}^{p}(\mathbf{x})$ is related to a local step flux, we can assume that its value vanishes away from $\mathbf{x}^{p}$ from the properties of the Green function (see expressions in 2D and 3D elastostatics in $[3,23])$ and can truncate its support. In that case, zero Dirichlet conditions can be prescribed over the boundary of $\mathcal{D}^{p}$.

Considering remarks 1 and 2, computing the transformation tensors will only require:

- Solving $P$ FEM problems over a small domain $\mathcal{D}^{p}$ implying $N_{x}^{s} \times N_{y}^{s}$ nodes, where $P$ is the number of components of $\mathcal{F}^{p}$ and where $N_{x}^{s} \times N_{y}^{s}$ can be much smaller than $N_{x} \times N_{y}$. For example, in the thermal case, there are $P=2$ independent components of $\mathcal{F}^{p}$ in $2 \mathrm{D}$, and $P=3$ in $3 \mathrm{D}$. In the elasticity case discussed in section 2.2, the step flux is replaced by a step eigenstress, having $P=3$ independent components in $2 \mathrm{D}, P=6$ in $3 \mathrm{D}$.

- Evaluating $\psi_{i j}^{m}(\mathbf{x}), \forall m \neq p$ will only require applying translation and periodicity conditions in the actual domain $\Omega$, by re-using the computed values of $\phi^{p j}(\mathbf{x})$. The computational details will be discussed in section 3 .

\subsection{Elastostatic problem}

In the case of elasticity, the localization problem reads as follows: given a macroscopic strain $\bar{\varepsilon}$, find the displacement field $\mathbf{u}(\mathbf{x})$ such that:

$$
\nabla \cdot(\mathbb{C}(\mathbf{x}): \varepsilon(\mathbf{x}))=0 \text { in } \Omega
$$

where $\mathbb{C}(\mathbf{x})$ is the fourth-order tensor of elastic properties and

$$
\varepsilon(\mathbf{u})=\frac{1}{2}\left(\nabla \mathbf{u}+\nabla \mathbf{u}^{T}\right)
$$




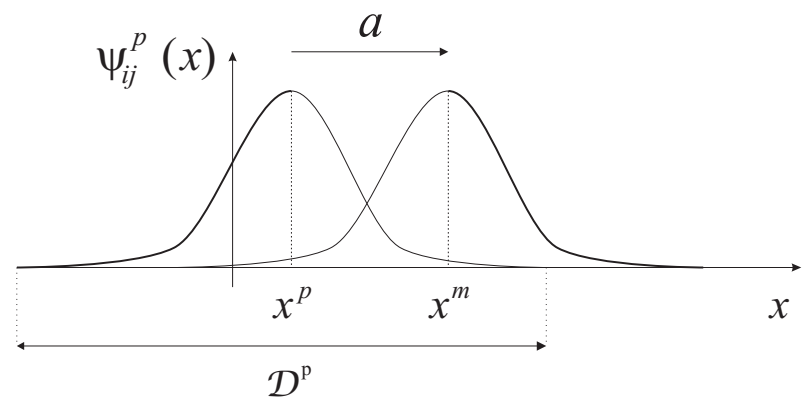

Figure 2. Translation of the function $\psi_{i j}^{p}(\mathbf{x})$ and its compact support $\mathcal{D}^{p}$.

verifying

$$
\langle\varepsilon(\mathbf{x})\rangle=\bar{\varepsilon} .
$$

Eq. (26) is satisfied for

$$
\varepsilon(\mathbf{x})=\bar{\varepsilon}+\tilde{\varepsilon},
$$

where $\tilde{\varepsilon}$ is a periodic fluctuation over $\Omega$. Introducing a reference medium with elastic properties $\mathbb{C}^{0}$ as

$$
\mathbb{C}(\mathbf{x})=\mathbb{C}^{0}+\mathbb{C}(\mathbf{x})-\mathbb{C}^{0},
$$

equation (24) becomes

$$
\nabla \cdot\left(\mathbb{C}^{0}: \varepsilon(\mathbf{x})\right)=-\nabla \cdot\left(\left[\mathbb{C}(\mathbf{x})-\mathbb{C}^{0}\right]: \varepsilon(\mathbf{x})\right) \text { in } \Omega .
$$

Using (27) we obtain

$$
\nabla \cdot\left(\mathbb{C}^{0}: \tilde{\varepsilon}(\mathbf{x})\right)=-\nabla \cdot(\hat{\boldsymbol{\tau}}(\varepsilon(\mathbf{x}))) \text { in } \Omega,
$$

with $\hat{\boldsymbol{\tau}}(\mathbf{x})=\left[\mathbb{C}(\mathbf{x})-\mathbb{C}^{0}\right]: \varepsilon(\mathbf{x})$. Then the Lippmann-Schwinger equation writes:

$$
\varepsilon(\mathbf{x})=\bar{\varepsilon}-\Gamma^{0} * \hat{\boldsymbol{\tau}}(\varepsilon(\mathbf{x}))
$$

where $\Gamma^{0}$ is the periodic Green function related to the linear operator in (30) (see Appendix 7).

We apply the same methodology as in the previous section and propose a discrete form of the convolution operator as:

$$
\tilde{\varepsilon}_{i j}^{n+1}(\mathbf{x})=\sum_{p} \psi_{i j k l}^{p}(\mathbf{x}) \hat{\tau}_{k l}^{n}\left(\mathbf{x}^{p}\right),
$$

where

$$
\psi_{i j k l}^{p}(\mathbf{x})=\varepsilon_{i j}\left(\phi^{p, k l}(\mathbf{x})\right) \equiv \varepsilon_{i j}^{p, k l}(\mathbf{x}) .
$$


In Eq. (33), $\phi^{p, k l}(\mathbf{x})$ is the displacement vector solution obtained by prescribing a constant unitary eigenstress $\mathcal{S}_{k l}^{p}(\mathbf{x})$ in a voxel centered on a point $\mathbf{x}^{p}$ with

$$
S_{i j}^{p, k l}(\mathbf{x})=\chi^{p}(\mathbf{x}) J_{i j}^{k l}(\mathbf{x})
$$

the second-order tensor $\mathbf{J}$ being defined in Appendix 7, Eq. (81).

In practice, for a $2 \mathrm{D}$ problem, 3 elementary eigenstress are prescribed at the point $\mathbf{x}^{p}$ :

$$
\mathcal{S}^{p, 11}(\mathbf{x})=\chi^{p}(\mathbf{x})\left[\begin{array}{ccc}
1 & 0 & 0 \\
0 & 0 & 0 \\
0 & 0 & 0
\end{array}\right], \quad \mathcal{S}^{p, 22}(\mathbf{x})=\chi^{p}(\mathbf{x})\left[\begin{array}{ccc}
0 & 0 & 0 \\
0 & 1 & 0 \\
0 & 0 & 0
\end{array}\right]
$$

and

$$
\mathcal{S}^{p, 12}(\mathbf{x})=\chi^{p}(\mathbf{x})\left[\begin{array}{lll}
0 & 1 & 0 \\
1 & 0 & 0 \\
0 & 0 & 0
\end{array}\right]
$$

The problem to be solved for computing $\psi_{i j k l}^{p}(\mathbf{x}) \equiv \varepsilon_{i j}^{p, k l}(\mathbf{x})$ is given by

$$
\nabla \cdot\left(\mathbb{C}^{0}: \varepsilon^{p, k l}(\mathbf{x})\right)=-\nabla \cdot\left(\mathcal{S}^{p, k l}(\mathbf{x})\right)
$$

with boundary conditions

$$
\phi^{p, k l}(\mathbf{x})=\mathbf{0} \text { on } \partial \mathcal{D}^{p}
$$

and where the indices $k, l$ are chosen to correspond to the elementary cases. Problem (37) can be easily solved by a finite element method, as described in section 3.2.

\subsection{Choice of the reference medium}

To ensure convergence of the iterative algorithm, Michel et al. [13] have shown that for an isotropic linear elastic medium, a sufficient condition is that

$$
\kappa^{0}>\max \{\kappa(x)\} / 2, \mu^{0}>\max \{\mu(x)\} / 2,
$$

where $\kappa$ and $\mu$ denote bulk and shear parameters. A similar condition for isotropic, linear, steady-state thermal problems can be derived as

$$
k^{0}>\max \{k(x)\} / 2 .
$$

Another interesting discussion on the convergence rate of iterative schemes can be found in [15]. A case of locally anisotropic materials has been treated in the context of FFT in Willot [25] for dealing with voids embedded in an anisotropic elastic matrix. 


\section{Computational details}

\subsection{Thermal problems}

3.1.1. Computation of transformation tensors by FEM: weak form In the present work, (20)(21) is solved by means of the Finite element method. The grid $N_{x}^{s} \times N_{y}^{s}$ related to $\mathcal{D}^{p}$ is used, and quadrilateral bilinear elements are constructed such as one element corresponds to a voxel in the material grid (see figure 1). The weak form associated to (20)-(21) is provided as follows. Multiplying (20) by a test function $\delta \phi$ and integrating over $\mathcal{D}^{p}$ gives:

$$
\int_{\mathcal{D}^{p}} k^{0}\left(\nabla \cdot\left(\nabla \phi^{p j}(\mathbf{x})\right)\right) \delta \phi(\mathbf{x}) d \Omega=-\int_{\mathcal{D}^{p}}\left(\nabla \cdot\left(\mathcal{F}^{j p}(\mathbf{x})\right)\right) \delta \phi(\mathbf{x}) d \Omega .
$$

Given a vector field $\mathbf{v}$ and a scalar field $u$ the property:

$$
u \nabla \cdot(\mathbf{v})=\nabla \cdot(u \mathbf{v})-\mathbf{v} \cdot \nabla \mathbf{u}
$$

is applied to (41) yielding

$$
\begin{aligned}
& \int_{\mathcal{D}^{p}} k^{0} \nabla \cdot\left(\nabla \phi^{p j}(\mathbf{x}) \delta \phi(\mathbf{x})\right) d \Omega-\int_{\mathcal{D}^{p}} k^{0} \nabla \boldsymbol{\phi}^{p j}(\mathbf{x}) \cdot \nabla \delta \phi(\mathbf{x}) d \Omega \\
& =-\int_{\mathcal{D}^{p}} \nabla \cdot\left(\mathcal{F}^{p j}(\mathbf{x}) \delta \phi(\mathbf{x})\right) d \Omega+\int_{\mathcal{D}^{p}} \mathcal{F}^{p j}(\mathbf{x}) \cdot \nabla \delta \phi(\mathbf{x}) d \Omega .
\end{aligned}
$$

Using the divergence theorem we obtain:

$$
\begin{aligned}
& \int_{\mathcal{D}^{p}} k^{0} \nabla \phi^{p j}(\mathbf{x}) \cdot \nabla \delta \phi(\mathbf{x}) d \Omega=-\int_{\mathcal{D}^{p}} \mathcal{F}^{p j}(\mathbf{x}) \cdot \nabla \delta \phi(\mathbf{x}) d \Omega \\
& +\int_{\partial \mathcal{D}^{p}} k^{0} \nabla \phi^{p j}(\mathbf{x}) \cdot \mathbf{n} \delta \phi(\mathbf{x}) d \Gamma+\int_{\partial \mathcal{D}^{p}} \mathcal{F}^{p j}(\mathbf{x}) \cdot \mathbf{n} \delta \phi(\mathbf{x}) d \Gamma,
\end{aligned}
$$

where $\partial \mathcal{D}^{p}$ denotes the boundary of $\mathcal{D}^{p}$. Taking $\delta \phi(\mathbf{x}) \in H_{0}^{1}\left(\mathcal{D}^{p}\right)$ and as $\phi^{p j}(\mathbf{x})$ also vanishes on $\partial \mathcal{D}^{p}$ we obtain the following weak form: find $\phi^{p j}(\mathbf{x}) \in H_{0}^{1}(\Omega)$ such that

$$
\int_{\mathcal{D}^{p}} k^{0} \nabla \phi^{p j}(\mathbf{x}) \cdot \nabla \delta \phi(\mathbf{x}) d \Omega=-\int_{\mathcal{D}^{p}} \mathcal{F}^{p j}(\mathbf{x}) \cdot \nabla \delta \phi(\mathbf{x}) d \Omega \quad \forall \delta \phi(\mathbf{x}) \in H_{0}^{1}(\Omega) .
$$

A classical FEM discretization of (45) leads to a linear system of equations in the form

$$
\mathbf{K} \mathbf{T}^{p j}=\mathbf{G}^{p j} .
$$

As a results, the temperature field $\phi^{p j}(\mathbf{x})$ is found at the nodes of the mesh (see figure 1 (a)) and is provided in vector $\mathbf{T}^{p j}$. The derivatives $\nabla_{i} \phi^{p j}(\mathbf{x}) \equiv \psi_{i j}^{p}(\mathbf{x})$ are evaluated at the center of elements to match the material grid using the FEM shape functions derivatives. To evaluate $\psi_{i j}^{m}(\mathbf{x})$ for $m \neq p$ on the material grid, we simply translate the values of the discrete solution $\psi_{i j}^{p}$ and apply some periodic conditions on the boundary of $\Omega$, as illustrated in figure 3. Figure 3 (a) provides the plot of the function $\psi_{11}^{p}(\mathbf{x})$ in Eq. (18) for a FEM nodal grid $N_{x}^{s} \times N_{y}^{s}$ $=20 \times 20$, resulting from solving the problem (46) with $k^{0}=1 \mathrm{~W} \cdot \mathrm{m}^{-1} \cdot \mathrm{K}^{-1}$ and $\overline{\mathbf{G}}=\mathbf{e}_{1}$. Figures 3 (b) illustrates the mapping of $\psi_{11}^{p}(\mathbf{x})$ on the material grid, containing more voxels. The different images correspond to the cases where periodic conditions are applied near the boundaries. 


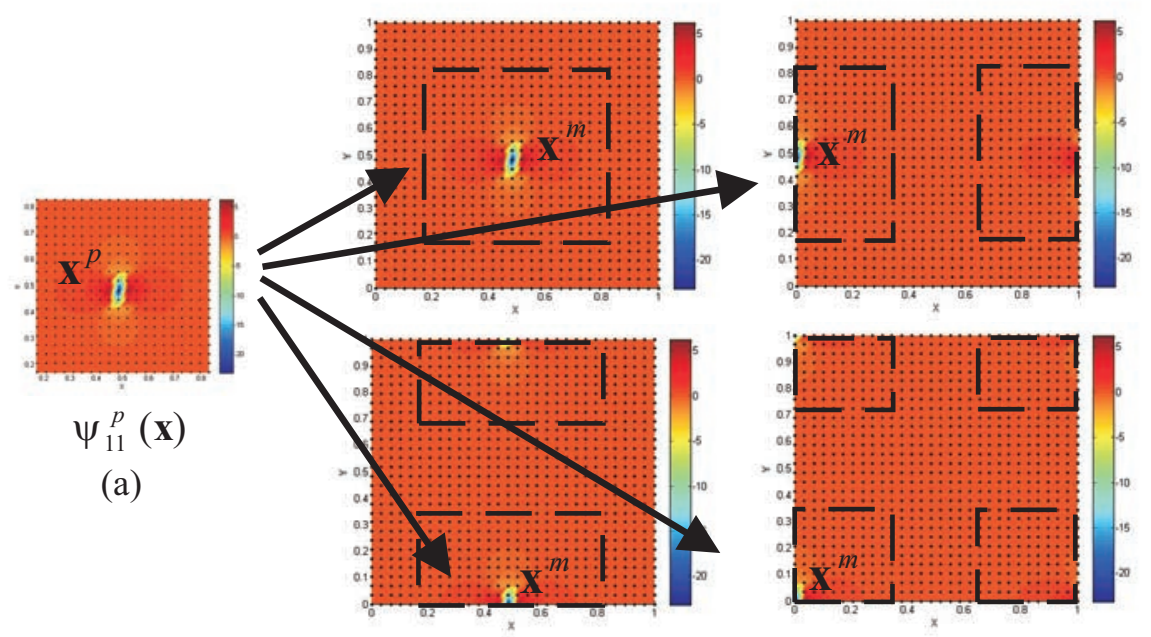

(b)

Figure 3. (a) Plot of the transformation tensor $\psi_{11}^{p}(\mathbf{x})$ of a reference point $\mathbf{x}^{p}$ computed on a $N_{x}^{s} \times N_{y}^{s}=20 \times 20$ grid; (b) projection of $\psi_{11}^{p}(\mathbf{x})$ on the $N_{x} \times N_{y}$ material grid for arbitrary points $\mathbf{x}^{m}$, illustrating the periodicity conditions to apply when points of the reference grid fall out of the material grid.

3.1.2. Algorithm The integral equation (12) can be solved iteratively using the scheme:

$$
\nabla T_{i}^{n+1}(\mathbf{x})=\overline{\mathbf{G}}-\Gamma_{i j}^{0} * \hat{q}_{j}\left(\nabla \mathbf{T}^{n}\right) .
$$

Replacing the convolution operator by its discrete counterpart in the form of spatial transformation tensors we obtain:

$$
\nabla T_{i}^{n+1}(\mathbf{x})=\overline{\mathbf{G}}+\sum_{p} \psi_{i j}^{p}(\mathbf{x}) \hat{q}_{j}^{n}\left(\mathbf{x}^{p}\right)
$$

Eq. (48) can be re-written in an alternative form by noting that for every compatible gradient field $\nabla \mathbf{T}$ (i.e. a vector field deriving from a temperature field), one has [13]:

$$
\Gamma^{0} *\left(\mathbf{k}^{0} \nabla \mathbf{T}\right)=\nabla \mathbf{T}-\overline{\mathbf{G}} .
$$

Introducing (49) in (47) yields the expression

$$
\nabla T_{i}^{n+1}(\mathbf{x})=\nabla T_{i}^{n}(\mathbf{x})-\Gamma_{i j}^{0} *\left(k(\mathbf{x}) \nabla \mathbf{T}^{n}(\mathbf{x})\right) .
$$

Replacing the convolution operator by its discrete form yields the following algorithm. Given $\overline{\mathbf{G}}, C$ a convergence criterion (see section 3.3 ) and $\delta$ a tolerance parameter:

1. Initialize $\nabla \mathbf{T}^{0}(\mathbf{x})=\overline{\mathbf{G}}$.

2. WHILE $C\left(\nabla \mathbf{T}^{n+1}\right)>\delta$

$$
\text { Compute } \nabla T_{i}^{n+1}(\mathbf{x})=\nabla T_{i}^{n}(\mathbf{x})+\sum_{p} \nabla \psi_{i j}^{p}(\mathbf{x})\left[k\left(\mathbf{x}^{p}\right) \nabla T_{j}^{n}\left(\mathbf{x}^{p}\right)\right] \text {. }
$$

3. Compute the new flux $q_{i}^{n+1}(\mathbf{x})=-k(\mathbf{x}) \nabla T_{i}^{n+1}(\mathbf{x})$.

4. Compute $C\left(\nabla \mathbf{T}^{n+1}\right)$.

5. Go to (2). 


\subsection{Elasticity}

3.2.1. Computation of transformation tensors by FEM: weak form Here we provide the weak form of the FEM problem used to compute the transformation tensors related to the convolution operator in the Lippmann-Schwinger equation for elastic problem. Multiplying (37) by a test function $\delta \phi \in H_{0}^{1}(\Omega)$, integrating over $\Omega$ and carrying out an integration by parts, the weak form related to (37)-(38) is given as follows. Find the displacements $\phi^{p, k l} \in H_{0}^{1}(\Omega)$ such that:

$$
\int_{\mathcal{D}^{p}} \varepsilon(\delta \phi(\mathbf{x})): \mathbb{C}^{0}: \varepsilon\left(\phi^{p, k l}(\mathbf{x})\right) d \Omega=-\int_{\mathcal{D}^{p}} \varepsilon(\delta \phi(\mathbf{x})): \mathcal{S}^{p, k l}(\mathbf{x}) d \Omega \quad \forall \delta \phi \in H_{0}^{1}(\Omega) .
$$

Introducing a FEM discretization of (51) leads to a discrete linear system in the form:

$$
\mathbf{K u}^{p, k l}=\mathbf{F}\left(\mathcal{S}^{p, k l}(\mathbf{x})\right),
$$

where $\mathbf{u}^{p, k l}$ is the solution vector containing the nodal values of $\phi^{p, k l}(\mathbf{x})$. The first step of the algorithm is then to solve (52) for the indices $k, l$ corresponding to the elementary cases defined in section 2.2. Then the functions $\psi_{i j k l}^{p}$ are computed according to (33) at the center of elements and stored.

3.2.2. Algorithm Considering similar arguments than in the thermal case and using the property of the Green operator [13]:

$$
\Gamma^{0} *\left(\mathbb{C}^{0}: \varepsilon\right)=\varepsilon-\bar{\varepsilon}
$$

we obtain the following algorithm, given $\bar{\varepsilon}$ :

1. Initialize $\varepsilon^{0}(\mathbf{x})=\bar{\varepsilon}, \boldsymbol{\sigma}^{0}(\mathbf{x})=\mathbb{C}(\mathbf{x}): \bar{\varepsilon}$.

2. WHILE $C\left(\varepsilon^{n+1}\right)>\delta$

Compute

$$
\varepsilon_{i j}^{n+1}(\mathbf{x})=\varepsilon_{i j}^{n}(\mathbf{x})+\sum_{p} \psi_{i j k l}^{p}(\mathbf{x}) \sigma_{k l}^{n}\left(\mathbf{x}^{p}\right) .
$$

3. Compute the stress $\boldsymbol{\sigma}^{n+1}(\mathbf{x})=\mathbb{C}(\mathbf{x}): \varepsilon^{n+1}(\mathbf{x})$.

4. Compute $C\left(\varepsilon^{n+1}\right)$.

5. Go to (2).

\subsection{Convergence criteria}

The following criteria have been investigated

$$
\begin{aligned}
& C_{1}=\frac{\left\|\boldsymbol{\sigma}^{n+1}(\mathbf{x})-\boldsymbol{\sigma}^{n}(\mathbf{x})\right\|}{\left\|\boldsymbol{\sigma}^{n+1}(\mathbf{x})\right\|} \\
& C_{2}=\frac{\left\|\mathbb{C}^{0}:\left(\varepsilon^{n+1}(\mathbf{x})-\varepsilon^{n}(\mathbf{x})\right)\right\|}{\left\|\boldsymbol{\sigma}^{n+1}(\mathbf{x})\right\|}=\frac{\left\|\mathbb{C}^{0}: \Gamma^{0} * \boldsymbol{\sigma}^{n}\right\|}{\left\|\boldsymbol{\sigma}^{n+1}(\mathbf{x})\right\|}
\end{aligned}
$$


and

$$
C_{3}=\frac{\left\|\left\langle\boldsymbol{\sigma}^{n+1}(\mathbf{x})\right\rangle-\left\langle\boldsymbol{\sigma}^{n}(\mathbf{x})\right\rangle\right\|}{\left\|\left\langle\boldsymbol{\sigma}^{n+1}(\mathbf{x})\right\rangle\right\|} .
$$

Criteria $C_{1}$ and $C_{2}$ are based on local quantities, while $C_{3}$ is based on macroscopic ones. In the above, $\Gamma^{0} * \sigma^{n}$ is understood as its discrete counterpart $\sum_{p} \psi_{i j k l}^{p} \sigma_{k l}^{n}$. In the thermal case, the strain and stress are simply replaced by the temperature gradient and flux, respectively.

\subsection{Remarks}

1. It is worth noting that the Green functions presented in Eqs (71) and (82) are not used directly in the present work. Instead, the functions $\psi$ defined in Eqs. (18) and (32) are approximations of the convolution of these Green functions with a unitary flux/eigenstress localized in one voxel. The result are provided directly by means of solving the finite element problems defined in Eqs. (45) and (52).

2. The structure and implementation of the algorithm are very simple. Furthermore, as all sums involved in Eq. (54) are independent, distributing them over many processors is direct. This point will be tested in the numerical examples of section 4.8.

3. The present algorithm employs the same basic scheme as in [10] but only operates in the real space domain. Then, any other variant could be investigated, like a stress approach accelerated schemes or algorithms for handling infinite contrasts or nonlinear problems $[17,13,15]$. These points would deserve future separated studies.

4. Memory requirements are very low, as no global rigidity matrix needs to be constructed neither stored, and scale linearly with the number of voxels.

5. The computational time for each iteration is linear with the number of voxels, and proportional to $N_{x}^{s} \times N_{y}^{s} \times V, V$ being the number of voxels. A deeper analysis on the computational times will be performed in section 4.8 .

\section{Numerical examples}

\subsection{Thermal problem: Influence of the reference medium and phase contrast on convergence}

In this first test the influence of the reference medium on the convergence is examined for the case of thermal conductivity. A $2 \mathrm{D}$ r.v.e composed of a square cell with a centered cylindrical inclusion is considered. The volume fraction is $f=0.3$. The conductivity of the matrix is $k_{\text {mat }}=$ $1 \mathrm{~W} \cdot \mathrm{m}^{-1} \cdot \mathrm{K}^{-1}$ and several values of inclusion's conductivity are investigated according to figure 4. A macroscopic gradient $\overline{\mathbf{G}}=\mathbf{e}_{1}$ is prescribed. The grid for computing the transformation tensors is chosen as $N_{x}^{s} \times N_{y}^{s}=80 \times 80$. For this first test, we also chose for the material grid $N_{x} \times N_{y}=80 \times 80$. The influence of $N_{x}^{s}$ on the convergence will be discussed in section 4.5. the functions $\psi_{i j}^{p}(\mathbf{x})$ are computed according to the procedure described in section 3.1. When the value of $k^{0}$ is changed the basis functions $\psi_{i j}^{p}(\mathbf{x})$ are simply scaled according to $\psi_{i j}^{p}\left(k^{0}, \mathbf{x}\right)=\psi_{i j}^{p}(1, \mathbf{x}) / k^{0}$. We then run the algorithm described in section 3.1. Here the criterion $C_{1}$ (Eq. (55)) is chosen. The number of iterations until convergence $\left(\delta=10^{-3}\right.$ in all following example) is plotted in figure 4 as a function of the ratio $k^{0} / \max (k(\mathbf{x}))$. We can note that the theoretical limit for convergence $k^{0} / \max (k(\mathbf{x}))>1 / 2$ is verified numerically. The optimal 


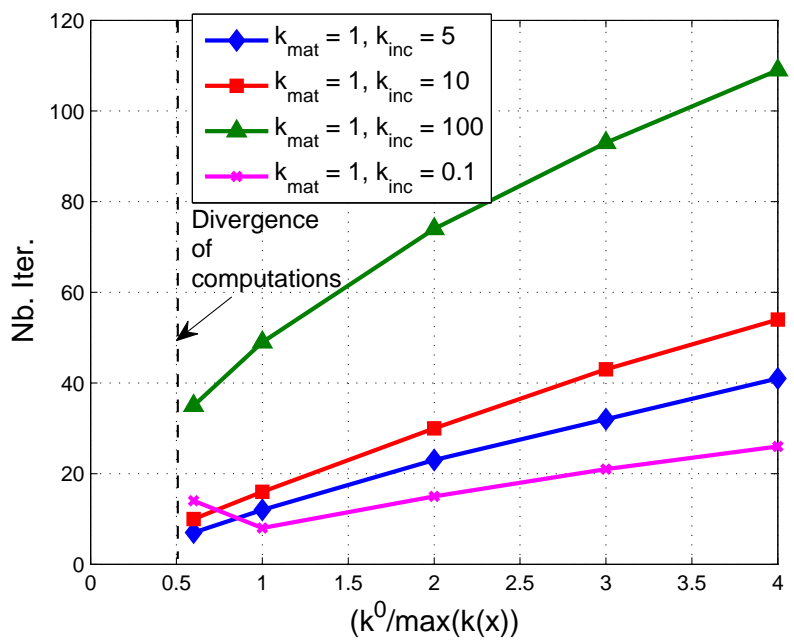

Figure 4. Influence of the reference medium on the convergence for the thermal problem (units in $\left.\mathrm{W} \cdot \mathrm{m}^{-1} \cdot \mathrm{K}^{-1}\right)$.

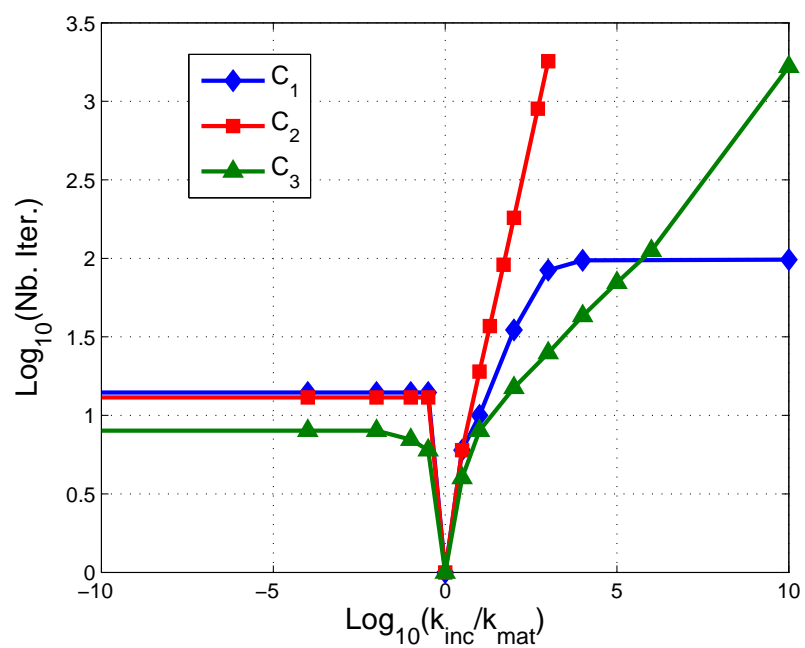

Figure 5. Thermal problem: Influence of the contrast of phase properties on the convergence of the algorithm.

value of $k^{0} / \max (k(\mathbf{x}))$ is found to be nearly above 0.5 . We will then chose in the next examples $k^{0} / \max (k(\mathbf{x}))=0.6$.

In this next test, the convergence is examined with respect to the contrast between phase properties in figure 5. The r.v.e., macroscopic gradient and grid sizes are the same as in the previous test. The different criteria (55), (56) and (57) are compared.

We can notice that according to the chosen criterion, the convergence can be strongly 


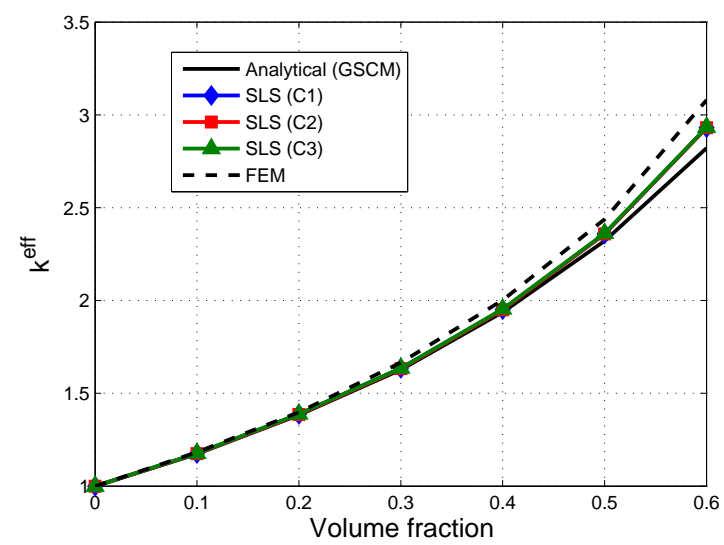

Figure 6. Effective conductivity versus volume fraction.

influenced by the contrast of the phases, which is a classical results in such iterative schemes $[17,13]$. We can note that when inclusions are perfectly conducting $\left(k_{i n c} / k_{\text {mat }}=10^{10}\right)$, both criteria $C_{1}$ and $C_{3}$ lead to a reasonable number of iterations $\left(10^{2}\right.$ and $10^{3}$, respectively), while criterion $C_{2}$ leads to divergence of the iterative scheme.

\subsection{Effective thermal conductivity}

The aim of this example is to evaluate the accuracy of the method for computing the effective thermal properties of a composite material. The r.v.e is the same as in the previous example. Assuming that the equivalent homogeneous material is transversally isotropic, the effective conductivity can be obtained by prescribing a macroscopic gradient $\overline{\mathbf{G}}=\mathbf{e}_{1}+\mathbf{e}_{2}$ and computing

$$
k^{e f f}=\left\langle k(\mathbf{x}) \nabla T_{1}(\mathbf{x})\right\rangle .
$$

where $\nabla T_{1}(\mathbf{x})$ is the $x$ - component of the temperature gradient solution at equilibrium. Firstly, the volume fraction $f$ is varied for a fixed contrast of phase properties $k_{\text {inc }}=10 \mathrm{~W} \cdot \mathrm{K}^{-1} \cdot \mathrm{m}^{-1}$, $k_{m a t}=1 \mathrm{~W} . \mathrm{K}^{-1} \cdot \mathrm{m}^{-1}$. We chose $N_{x}^{s} \times N_{y}^{s}=80 \times 80$ and $N_{x} \times N_{y}=80 \times 80$. We compare the obtained results with an analytical solution provided by a generalized self consistent model:

$$
k^{e f f}=k_{m a t}+\frac{D \cdot k_{m a t} \cdot f\left[k_{i n c}-k_{m a t}\right]}{D \cdot k_{m a t}+(1-f)\left[k_{i n c}-k_{m a t}\right]},
$$

where $D$ is the space dimension, in figure 6 . We can note a good accuracy for all the range of volume fractions. We also note that the different criteria are quantitatively equivalent in this case.

Secondly, the volume fraction is kept constant to $f=0.4$ while the contrast changes, fixing $k_{m a t}$ as $1 \mathrm{~W} \cdot \mathrm{K}^{-1} \cdot \mathrm{m}^{-1}$. Here again, a very good accuracy is appreciated for all the considered contrast ranges, as shown in figure 7 , and for all criteria. We compare in table I the number of iterations required to achieve convergence for each criterion and the corresponding numerical values. It appears that for all contrasts, using criterion $C_{3}$ based on the macroscopic values of stress is much faster and leads to a comparable accuracy regarding effective properties. 


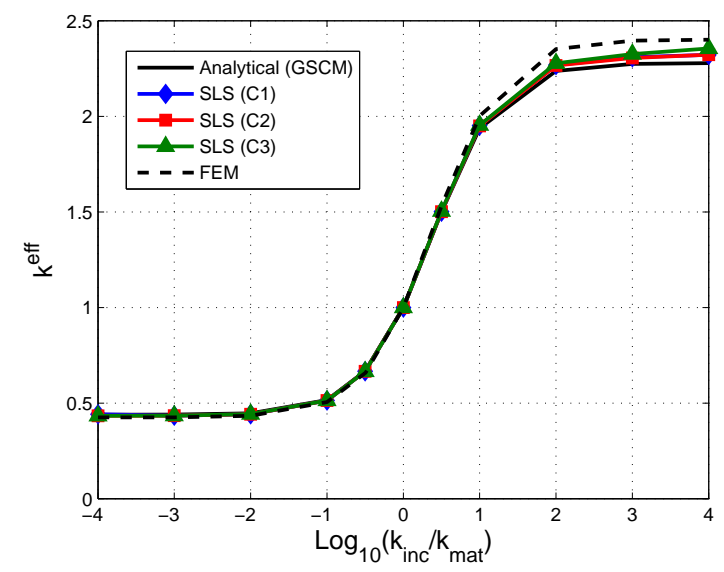

Figure 7. Effective conductivity versus contrast of phase properties for a fixed volume fraction.

Table I. Thermal effective properties: comparison of results obtained by the different criteria with respect to the number of iterations.

\begin{tabular}{lllllllll}
\hline Contrast & $\begin{array}{l}C_{1} \\
k^{\text {eff }}\end{array}$ & $\mathrm{Nb}$. iter & $k^{\text {eff }}$ & $\mathrm{Nb}$. iter & $k^{\text {eff }}$ & Nb. iter & GSCM & FEM \\
\hline $10^{-4}$ & 0.442 & 13 & 0.434 & 12 & 0.434 & 6 & 0.439 & 0.424 \\
$10^{-3}$ & 0.434 & 13 & 0.434 & 12 & 0.435 & 6 & 0.440 & 0.425 \\
$10^{-2}$ & 0.442 & 13 & 0.442 & 12 & 0.442 & 6 & 0.447 & 0.4331 \\
$10^{-1}$ & 0.513 & 13 & 0.513 & 11 & 0.514 & 6 & 0.516 & 0.505 \\
1 & 1 & 1 & 1 & 1 & 1 & 1 & 1 & 1 \\
10 & 1.951 & 8 & 1.950 & 10 & 1.954 & 10 & 1.937 & 2.00 \\
$10^{2}$ & 2.266 & 31 & 2.266 & 154 & 2.278 & 26 & 2.238 & 2.395 \\
$10^{3}$ & 2.309 & 88 & 2.305 & 1258 & 2.325 & 26 & 2.275 & 2.400 \\
\hline
\end{tabular}

\subsection{Thermal problem: local fields in a microstructure with 1000 inclusions}

An example involving 1000 inclusions is carried out. The r.v.e. consists in a square unit cell containing 1000 cylindrical inclusions whose positions are provided by an uniform probability distribution. Penetration between inclusions is tolerated. The volume fraction of inclusions is chosen as $f=0.2$ which gives a radius $R=7.9788$ voxels. The phase properties are $k_{\text {inc }}=10$ $\mathrm{W} \cdot \mathrm{K}^{-1} \cdot \mathrm{m}^{-1}, k_{m a t}=1 \mathrm{~W} \cdot \mathrm{K}^{-1} \cdot \mathrm{m}^{-1}$. A macroscopic gradient $\overline{\mathbf{G}}=\mathbf{e}_{1}+\mathbf{e}_{2}$ is prescribed. We chose $N_{x}^{s} \times N_{y}^{s}=80 \times 80$. In that case, the size of the grid used to compute the transformation tensors is much smaller than the material grid, chosen as $N_{x} \times N_{y}=1000 \times 1000$. The algorithm converges in 7 iterations. Local field distribution is plotted in figure 9. Discussion on CPU times and parallel computing is further developed in section 4.8. 


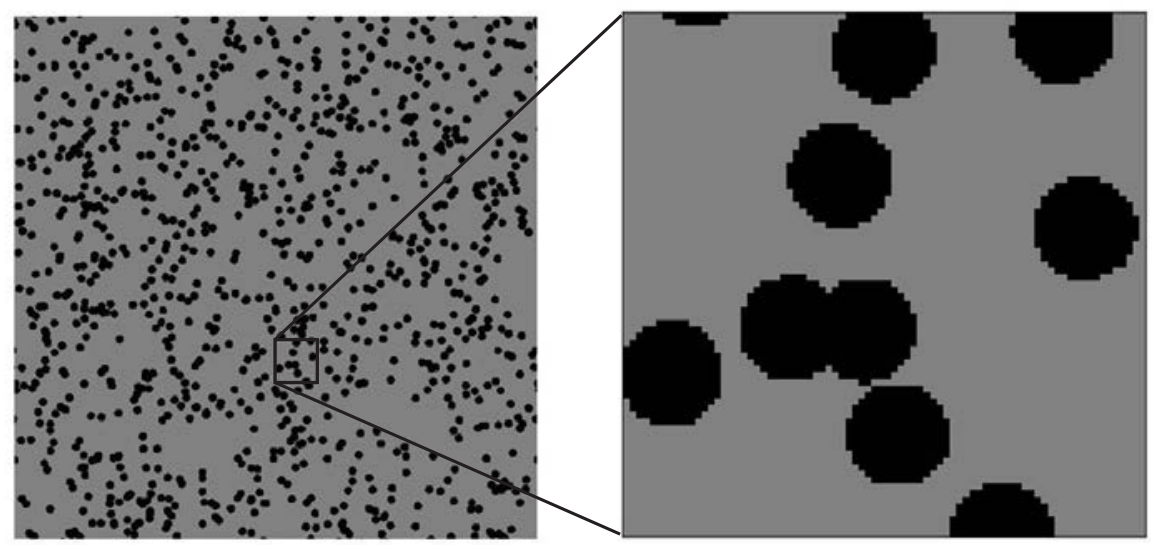

Figure 8. Microstructure with 1000 inclusions, $1000 \times 1000$ grid. The zoom zone refers to a $80 \times 80$ grid.

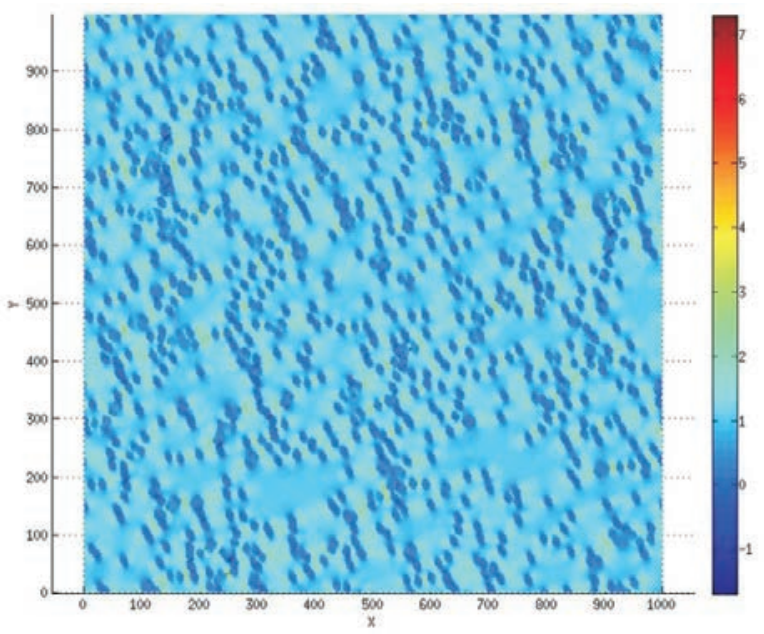

Figure 9. Microstructure with 1000 inclusions, $1000 \times 1000$ grid, $\nabla T_{1}(\mathbf{x})$ field.

\subsection{Elasticity: Influence of the reference medium and phase contrast}

The next series of tests concerns elasticity problems. In all further elasticity examples, plane strain is assumed and the phases are supposed to be characterized by an isotropic linear elastic behavior. We first test the influence of the reference medium on the convergence of the algorithm. The r.v.e. is a unit cell with a centered cylindrical inclusion. The volume fraction is chosen as $f=0.3$. The Lamé's parameters of the matrix are taken as $\mu_{m a t}=1 \mathrm{MPa}$ and $\lambda_{\text {mat }}=1 \mathrm{MPa}$, corresponding respectively to a Poisson's coefficient $\nu_{\text {mat }}=0.25$ and a Young's modulus $E=2.5 \mathrm{MPa}$. The parameters of the inclusions $\mu_{i n c}$ and $\lambda_{\text {inc }}$ vary. We took for all cases $\lambda=\mu$ corresponding to $\nu=0.25$ and $E=2.5 \lambda$. A macroscopic strain $\bar{\varepsilon}_{11}=1, \bar{\varepsilon}_{22}=0$, 


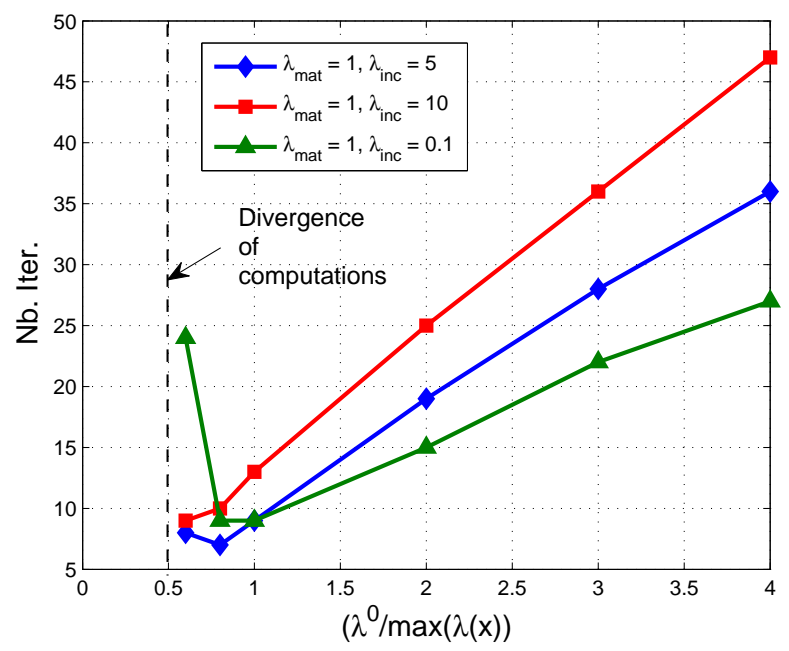

Figure 10. Elasticity: influence of the reference medium parameters on the convergence of the algorithm.

$\bar{\varepsilon}_{12}=0$ is prescribed. A grid with $N_{x}^{s}=N_{y}^{s}=80$ is chosen to compute the transformation tensors. The material grid is also taken as $80 \times 80$ in this test. We first analyze the influence of the choice of the reference medium characterized by $\lambda^{0} / \max (\lambda(\mathbf{x}))$ on the convergence. When $\lambda^{0}=\mu^{0}$ the functions can be simply scaled from $\psi_{i j k l}^{p}\left(\lambda^{0}\right)=\psi_{i j k l}^{p}(\lambda=1) / \lambda^{0}$. Otherwise, the functions $\psi_{i j k l}^{p}$ must be re-computed for each new set of $\lambda^{0}, \mu^{0}$, which is not penalizing as the transformation tensors are computed on a small grid. The criterion $C_{1}$ was chosen. Results are presented in figure 10. As in the thermal case, the optimal value of $\lambda^{0} / \max (\lambda(\mathbf{x}))$ is slightly above 0.5 , the theoretical limit for convergence predicted by (39) is numerically verified.

In a second test, we vary the contrast between phases $\lambda_{\text {inc }} / \lambda_{\text {mat }}$ for $\lambda^{0}=0.6 \max (\lambda(\mathbf{x}))$ and for the different criteria $C_{1}, C_{2}$ and $C_{3}$. All other parameters are the same as in the previous case. Results are presented in figure 11 . We can note a similar dependence to the contrast as in FFT algorithms [17] for all criteria. We note that the criterion $C_{3}$, based on macroscopic quantities, leads to a fastest convergence, as expected. The criterion $C_{2}$ leads to the highest number of iterations.

\subsection{Influence of $N_{x}^{s}$ on the convergence}

To maintain the computation of the transformation tensors in a reduced domain as compared to the size of the material grid, the numerical functions are truncated at a given distance from the reference point $\mathbf{x}^{p}$, where it is assumed that they vanish. In this test, we evaluate the influence of the size of the reduced domain, in other words $N_{x}^{s}$ if a square grid $N_{x}^{s} \times N_{x}^{s}$ is employed. We use different r.v.e. containing cylindrical inclusions whose centers are located on a square lattice. We define the characteristic fluctuation length (in voxels) $L_{\text {fluct }}$ as the number of voxels along one spatial direction in one pattern. When $L_{\text {fluct }}>N_{x}^{s}$, the r.v.e. contains a single inclusion. The volume fraction is $f=0.3$ and the material properties are $\lambda_{i n c}=\mu_{i n c}=10 \mathrm{MPa}, \lambda_{m a t}=\mu_{m a t}=1 \mathrm{MPa}$. A macroscopic strain $\bar{\varepsilon}_{11}=1, \bar{\varepsilon}_{22}=0, \bar{\varepsilon}_{12}=0$ 


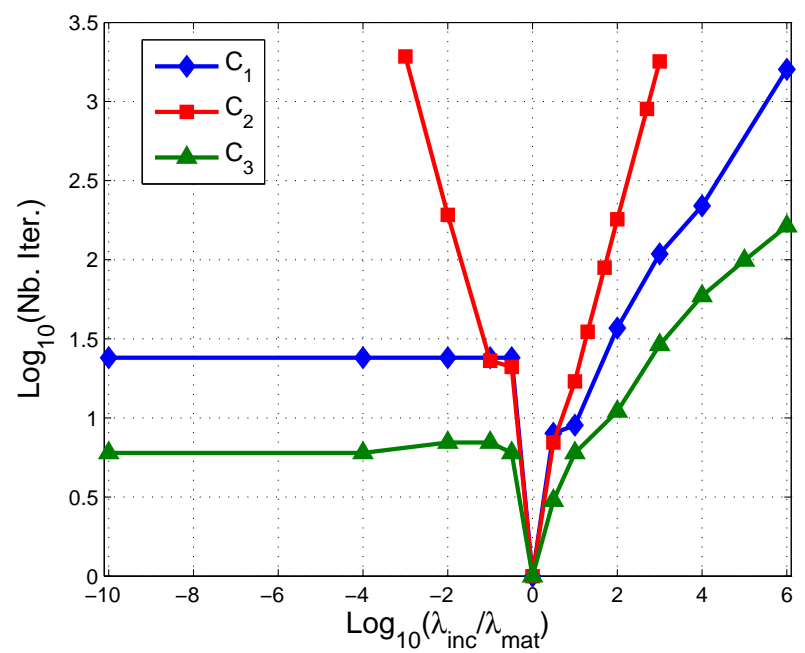

Figure 11. Elasticity: influence of the contrast of phase properties on the convergence of the algorithm.

is prescribed. We present in figure 12 the number of iterations to convergence for the different values of $N_{x}^{s}$ with respect to $L_{\text {fluct }}$. What appears is that if $L_{\text {fluct }} \leq N_{x}^{s}$, the convergence is not much affected by the choice of $N_{x}^{s}$. However, when $L_{\text {fluct }}>N_{x}^{s}$, the convergence is slower. This can be explained by the compact support of the transformation tensors. Even though their values are very small away from the reference voxel $p$, they are not zero. Then a knowledge of the characteristic fluctuation length (in voxel) might be useful to adapt $N_{x}^{s}$ using e.g. an estimated correlation length. Furthermore, it is worth noting that in the present work, the resolution for the transformation grids matches the ones of the microstructure grid. It could be possible to consider a transformation grid with a different resolution than the material grid. However, in that case the projection of the transformation data on the material grid would imply additional modifications of the algorithm that have not been considered here. These points should be investigated in future works.

\subsection{Elasticity: effective properties}

In this example we evaluate the accuracy of the method for computing the effective elastic properties of the composite. The considered r.v.e is a rectangular cell containing cylindrical inclusions whose centers are positioned on the vertices of a hexagonal lattice. An illustration of the corresponding voxel grid is depicted in figure 13. It has been shown in He and Zheng [5] that if the r.v.e. possesses a $C_{3}$ symmetry, then the effective behavior is transversally isotropic with in-plane properties characterized by elastic parameters $\mu^{e f f}$ and $\lambda^{e f f}$. These values are computed numerically as follows. Prescribing a macroscopic strain $\bar{\varepsilon}_{11}=0, \bar{\varepsilon}_{22}=0, \bar{\varepsilon}_{12}=1 / 2$, the effective shear coefficient is given by

$$
\mu^{e f f}=\left\langle\sigma_{12}(\mathbf{x})\right\rangle
$$




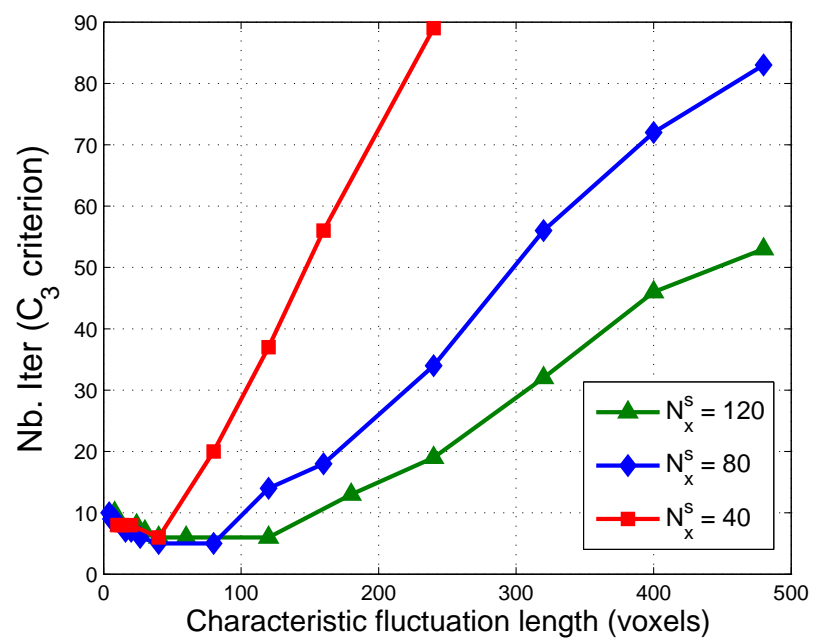

Figure 12. Influence of truncating the support of the transformation tensors on the convergence. The values of $N_{x}^{s}$ are given in voxels.

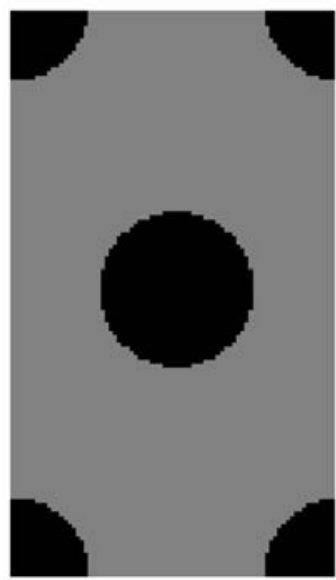

Figure 13. r.v.e. model corresponding to a hexagonal lattice, $f=0.2$

In the context of $2 \mathrm{D}$ plane strains, the transverse bulk modulus is found numerically by prescribing a strain $\bar{\varepsilon}_{11}=1 / 2, \bar{\varepsilon}_{22}=1 / 2, \bar{\varepsilon}_{12}=0$ and by computing

$$
\kappa^{e f f}=\frac{1}{3}\left\langle\sigma_{11}(\mathbf{x})+\sigma_{22}(\mathbf{x})+\sigma_{33}(\mathbf{x})\right\rangle,
$$

where $\sigma_{33}(\mathbf{x})=\lambda(\mathbf{x})\left(\varepsilon_{11}(\mathbf{x})+\varepsilon_{22}(\mathbf{x})\right)$. In both case, we compute the effective parameters and compare them with a FEM solution based on the same grid of voxels, where 4 nodes elements corresponding to each voxel are employed. Firstly, a fixed contrast of material properties within 


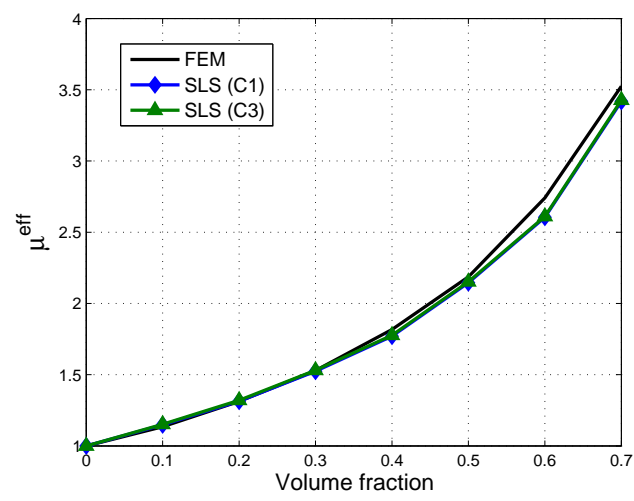

(a)

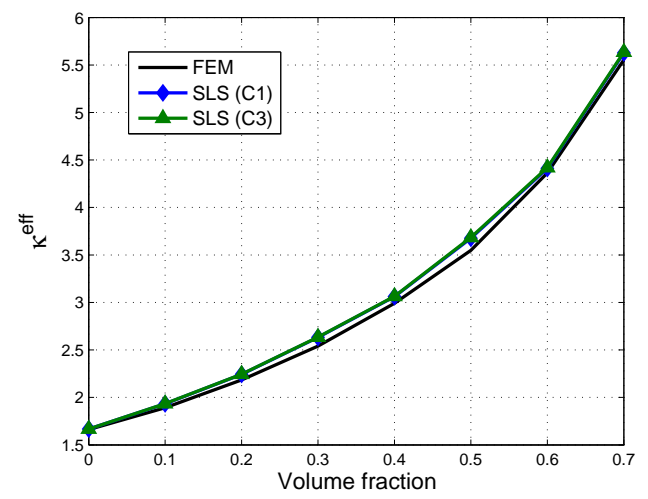

(b)

Figure 14. Comparison of effective elastic parameters computed with SLS and FEM for different volume fractions: (a) Effective shear coefficient $\mu^{\text {eff }}$ and (b) Effective transverse bulk modulus $\kappa^{\text {eff }}$.

Table II. Effective shear modulus $\mu^{e f f}$ : comparison of results obtained by SLS and FEM.

\begin{tabular}{llllll}
\hline Contrast & SLS $\left(C_{1}\right)$ & Nb. iter. $\left(C_{1}\right)$ & SLS $\left(C_{3}\right)$ & Nb. iter. $\left(C_{3}\right)$ & FEM \\
\hline $10^{-4}$ & 0.271 & 32 & 0.273 & 12 & 0.296 \\
$10^{-3}$ & 0.272 & 31 & 0.274 & 12 & 0.298 \\
$10^{-2}$ & 0.285 & 31 & 0.287 & 11 & 0.310 \\
$10^{-1}$ & 0.400 & 19 & 0.401 & 9 & 0.418 \\
1 & 1.000 & 1 & 1.000 & 1 & 1.000 \\
10 & 1.770 & 18 & 1.776 & 8 & 1.817 \\
$10^{2}$ & 1.988 & 54 & 2.011 & 21 & 2.047 \\
$10^{3}$ & 2.021 & 193 & 2.055 & 51 & 2.065 \\
$10^{4}$ & 2.029 & 476 & 2.071 & 85 & 2.065 \\
\hline
\end{tabular}

the different phases is chosen as $\lambda_{i n c}=10 \mathrm{MPa}, \mu_{i n c}=10 \mathrm{MPa}, \lambda_{\text {mat }}=1 \mathrm{MPa}, \mu_{\text {mat }}=1$ $\mathrm{MPa}$ while the volume fraction is changed. To show that the method is accurate even for small grids, we chose $N_{x}^{s}=N_{y}^{s}=40$ while the material grid is composed of $40 \times 69$ voxels. Results are provided in figure 14 (a)-(b) for criteria $C_{1}$ and $C_{3}$. We can note a good accuracy of the results with respect to the FEM solution. We also note that both $C_{3}$ and $C_{1}$ criteria give comparable results regarding the accuracy, while the $C_{3}$ criterion greatly alleviates the number of iterations. This point will be further detailed in the following.

Next the volume fraction is kept fixed to $f=0.4$ and the contrast is changed, keeping $\lambda_{\text {mat }}$ $=1 \mathrm{MPa}, \mu_{m a t}=1 \mathrm{MPa}$. Results are provided in figures 15 (a) - (b). Here again, a good agreement is observed with the FEM reference solution, even though the material grid was very coarse.

Next we indicate in Tables II and III the number of iterations used for both convergence criteria $C_{1}$ and $C_{3}$ and the corresponding values of effective parameters. We can note that regarding effective properties, the criterion $C_{3}$ gives a reasonable accuracy at much lower cost. 


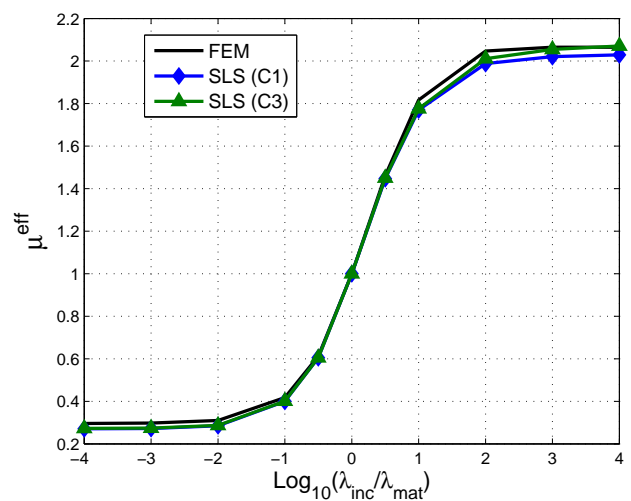

(a)

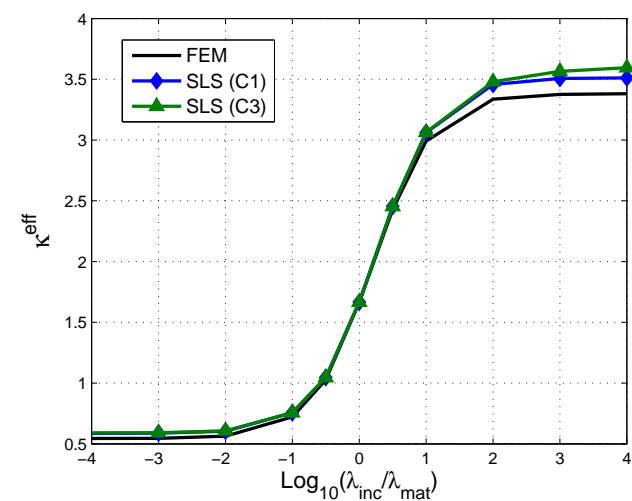

(b)

Figure 15. Comparison of effective elastic parameters computed with SLS and FEM for fixed volume fractions and varying contrast: (a) Effective shear coefficient $\mu^{\text {eff }}$ and (b) Effective transverse bulk modulus $\kappa^{e f f}$.

Table III. Effective bulk modulus $\kappa^{e f f}$ : comparison of results obtained by SLS and FEM.

\begin{tabular}{llllll}
\hline Contrast & SLS $\left(C_{1}\right)$ & Nb. iter. $\left(C_{1}\right)$ & SLS $\left(C_{3}\right)$ & Nb. iter. $\left(C_{3}\right)$ & FEM \\
\hline $10^{-4}$ & 0.586 & 20 & 0.590 & 8 & 0.544 \\
$10^{-3}$ & 0.588 & 19 & 0.591 & 8 & 0.545 \\
$10^{-2}$ & 0.604 & 17 & 0.607 & 7 & 0.563 \\
$10^{-1}$ & 0.756 & 13 & 0.756 & 6 & 0.722 \\
1 & 1.667 & 1 & 1.667 & 1 & 1.667 \\
10 & 3.061 & 12 & 3.064 & 7 & 2.992 \\
$10^{2}$ & 3.457 & 33 & 3.480 & 15 & 3.336 \\
$10^{3}$ & 3.507 & 121 & 3.565 & 37 & 3.376 \\
$10^{4}$ & 3.511 & 333 & 3.595 & 67 & 3.381 \\
\hline
\end{tabular}

\subsection{Elasticity: local fields}

We examine the accuracy of the local field distribution obtained by the SLS method in an elastic r.v.e.. Firstly, the r.v.e used in the previous example is considered with volume fraction $f=0.2$. The material parameters of phases are $\lambda_{\text {inc }}=10 \mathrm{MPa}, \mu_{\text {inc }}=10 \mathrm{MPa}, \lambda_{\text {mat }}=1 \mathrm{MPa}$, $\mu_{\text {mat }}=1 \mathrm{MPa}$. The macroscopic strain is $\bar{\varepsilon}_{11}=1, \bar{\varepsilon}_{22}=0, \bar{\varepsilon}_{12}=0$. We chose $N_{x}^{s}=N_{y}^{s}=80$. The material grid is composed of $80 \times 138$ voxels and the $C_{3}$ criterion is chosen to stop the iterative scheme, which converges in 8 iterations for $\delta=10^{-3}$ in that case. The local strain and stress fields are computed for both SLS and a FEM solution utilizing a mesh of 4 nodes elements matching the voxels. Results are presented in figures 16-23. Let $H$ denotes the dimension of the r.v.e. along $y$. Figures 16 and 17 depict the strain and stress solutions along a line $(x, y=H / 2)$ (passing through the central inclusion) and a line $(x, y=3 H / 4)$ passing between the inclusions. We can note that the local fields are accurately captured, even though a macroscopic criterion $C_{3}$ has been chosen.

Next, the microstructure is characterized by a more complex r.v.e. composed of 200 randomly 


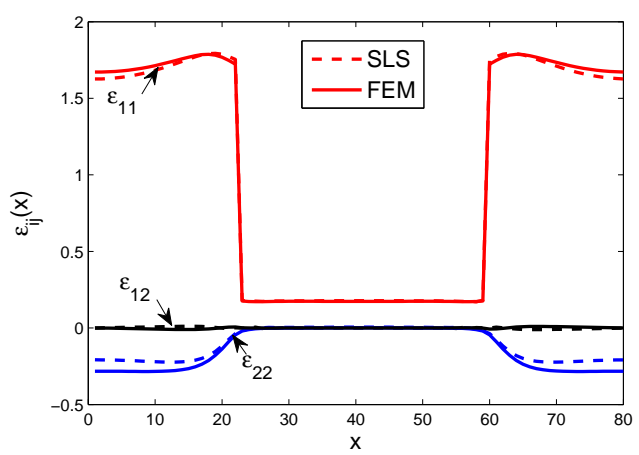

(a)

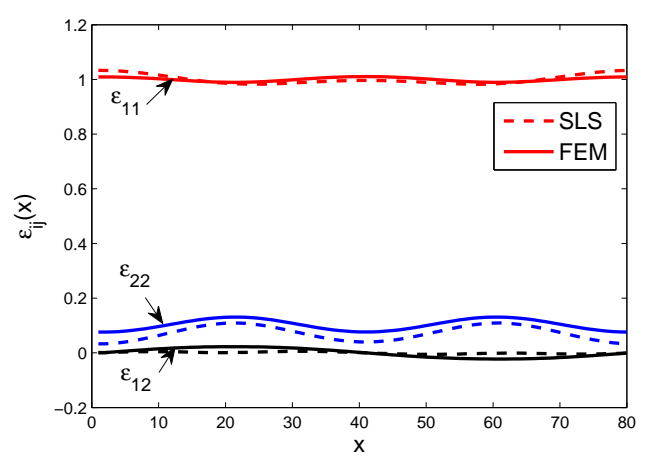

(b)

Figure 16. Strain solution in the hexagonal r.v.e. along a line (a) $y=H / 2$ and (b) $y=3 H / 4$.

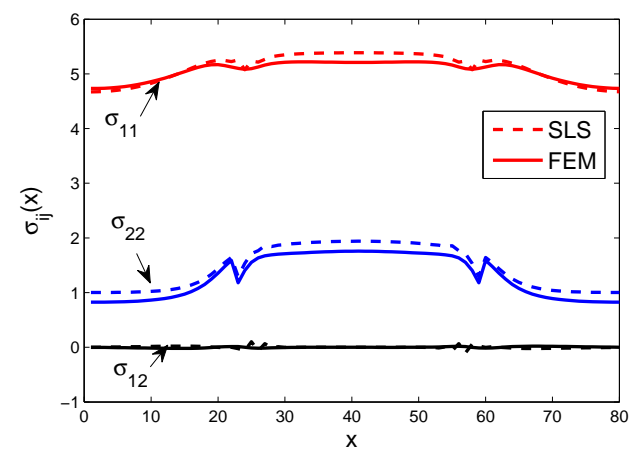

(a)

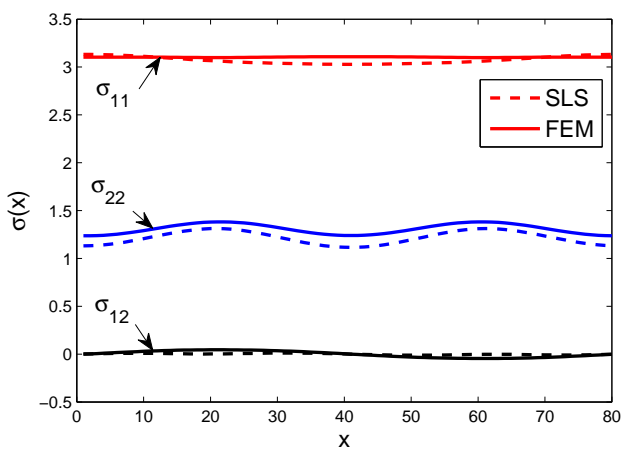

(b)

Figure 17. Stress solution in the hexagonal r.v.e. along a line (a) $y=H / 2$ and (b) $y=3 H / 4$.

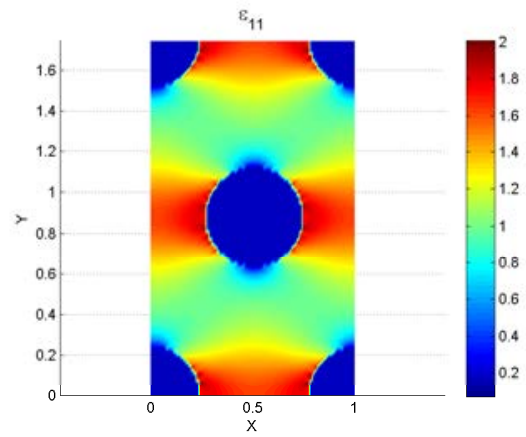

(a)

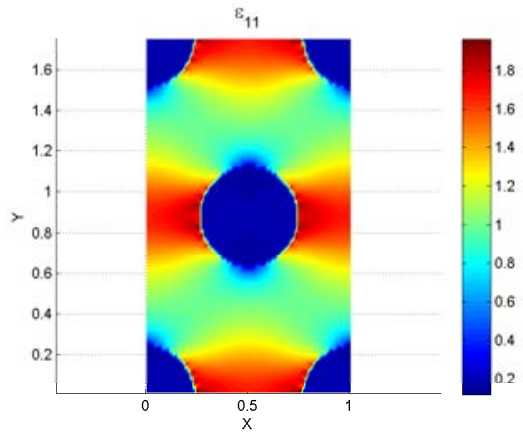

(b)

Figure 18. Strain distribution ( $\varepsilon_{11}$-component) in the r.v.e. based on hexagonal lattice: (a) SLS and (b) FEM solutions. 


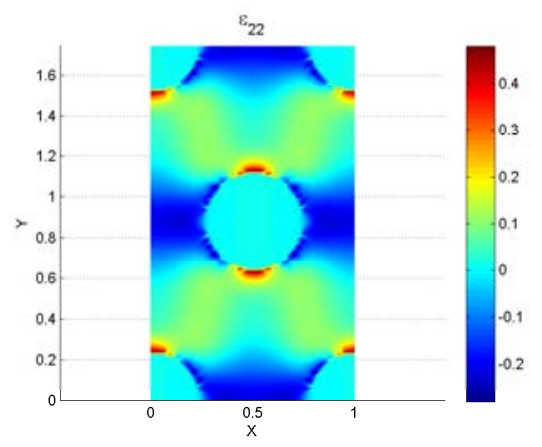

(a)

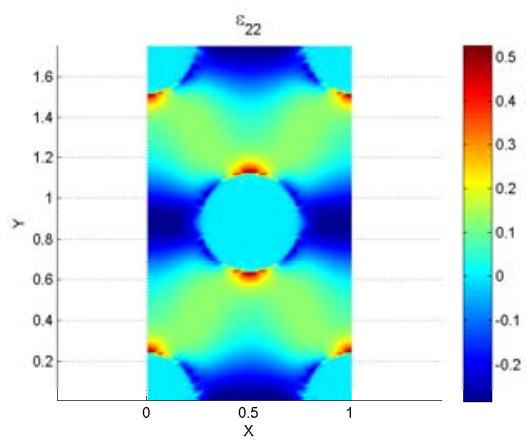

(b)

Figure 19. Strain distribution ( $\varepsilon_{22}$-component) in the r.v.e. based on hexagonal lattice: (a) SLS and (b) FEM solutions.

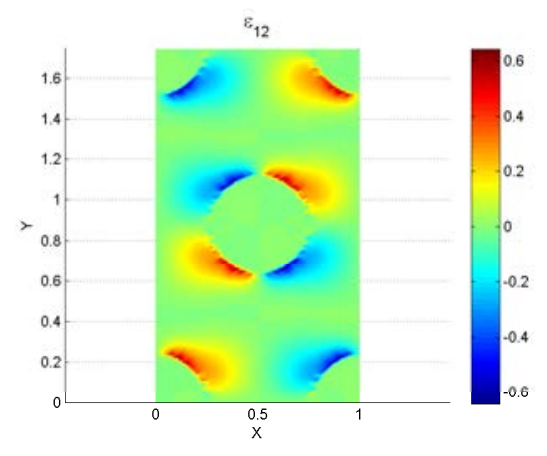

(a)

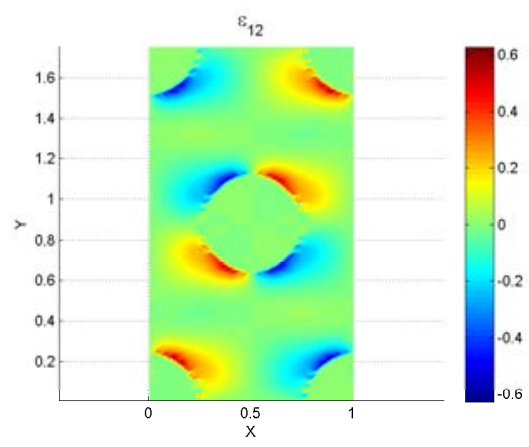

(b)

Figure 20. Strain distribution ( $\varepsilon_{12}$-component) in the r.v.e. based on hexagonal lattice: (a) SLS and (b) FEM solutions.

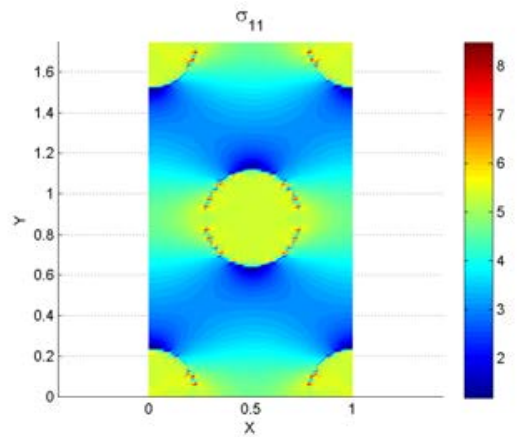

(a)

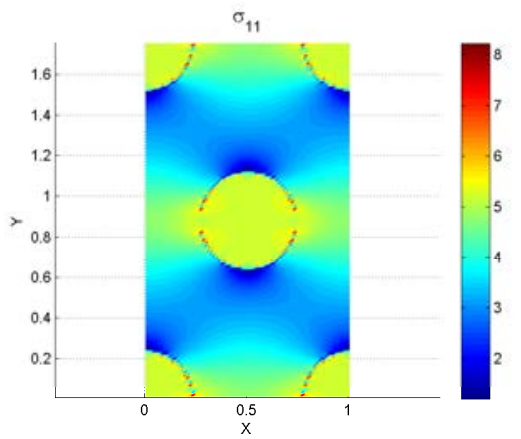

(b)

Figure 21. Stress distribution ( $\sigma_{11}$-component) in the r.v.e. based on hexagonal lattice: (a) SLS and (b) FEM solutions. 


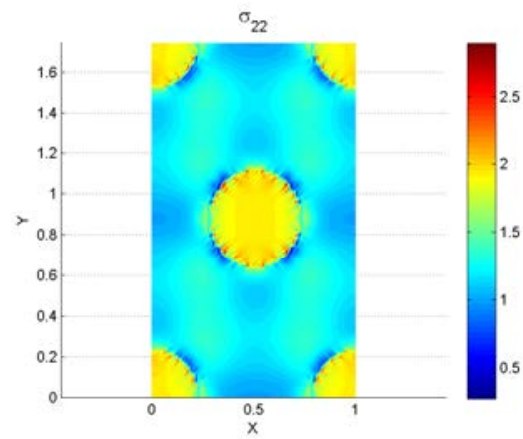

(a)

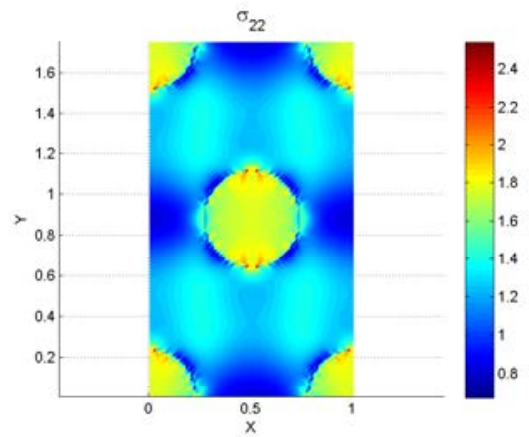

(b)

Figure 22. Stress distribution ( $\sigma_{22}$-component) in the r.v.e. based on hexagonal lattice: (a) SLS and (b) FEM solutions.

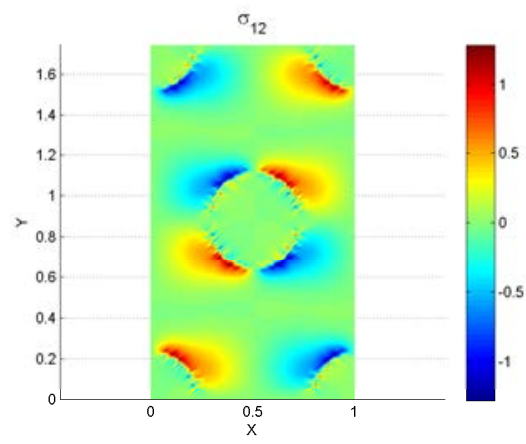

(a)

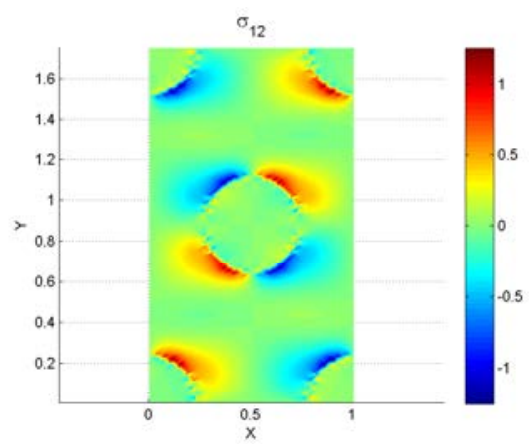

(b)

Figure 23. Stress distribution ( $\sigma_{12}$-component) in the r.v.e. based on hexagonal lattice: (a) SLS and (b) FEM solutions.

distributed cylindrical pores and a volume fraction $f=0.4$. The material properties are chosen as $\lambda_{i n c}=\mu_{i n c}=10^{-6} \mathrm{MPa}, \lambda_{\text {mat }}=\mu_{\text {mat }}=1 \mathrm{MPa}$. A macroscopic strain $\bar{\varepsilon}_{11}=1 / 2, \bar{\varepsilon}_{22}=1 / 2$, $\bar{\varepsilon}_{12}=0$ is prescribed. The material grid contains $300 \times 300$ voxels. The criterion $C_{3}$ is used to stop the computations. Comparison of von Mises stress fields obtained by SLS and FEM is provided in figures 24 and 25 . Due to high stress concentrations, the contrast plot is low in figure 24. By thresholding the maximum value in the color scale to $4 \mathrm{MPa}$, the local fields appear more clearly and can be compared between both methods in figure 25 . Here again, we can note that even though the macroscopic $C_{3}$ criterion was used, the local fields are in very good agreement with the reference (FEM) solution.

\subsection{Computational times}

In this example, we test the efficiency of the SLS algorithm for a problem involving a large number of degrees of freedom. A domain is defined in a $20000^{2}$ voxels grid, containing 400000 circular inclusions, whose centers coordinates are defined by a uniform probability law. The 


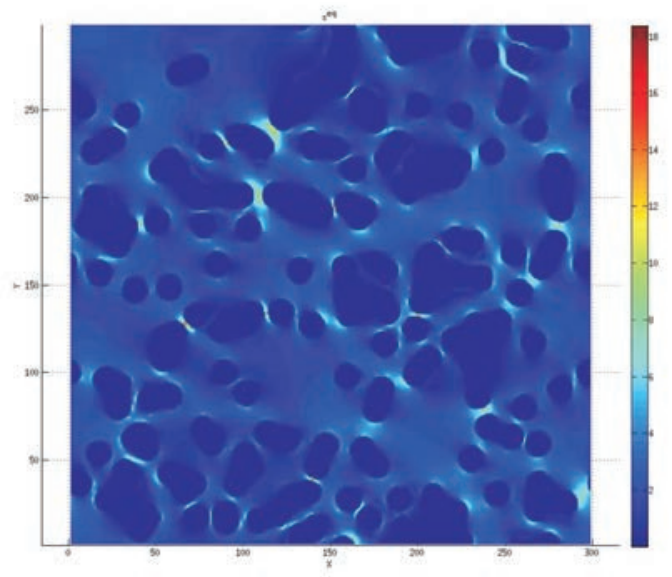

(a)

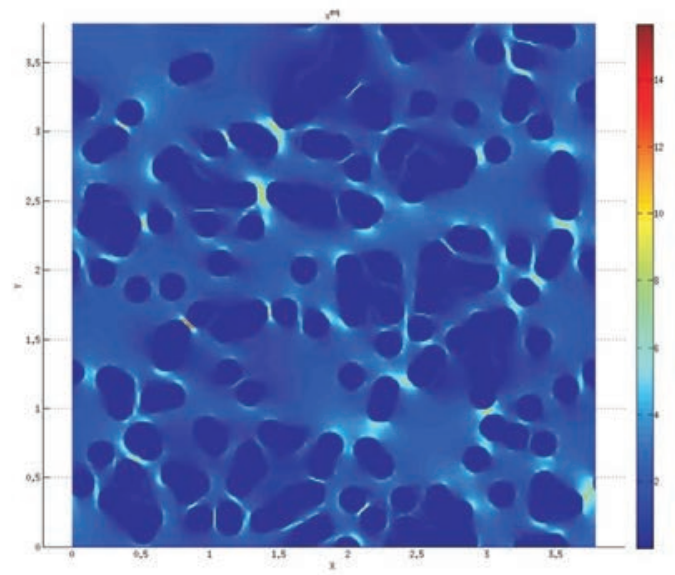

(b)

Figure 24. von Mises stress distribution in the r.v.e. with porous microstructure: (a) SLS and (b) FEM.

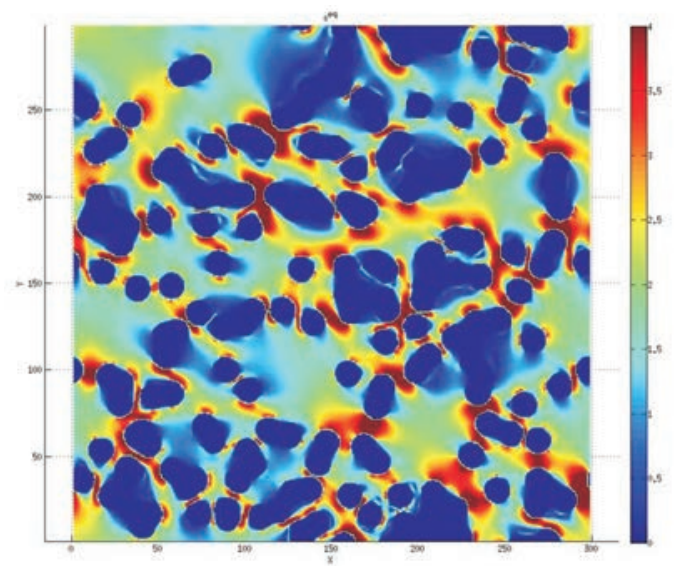

(a)

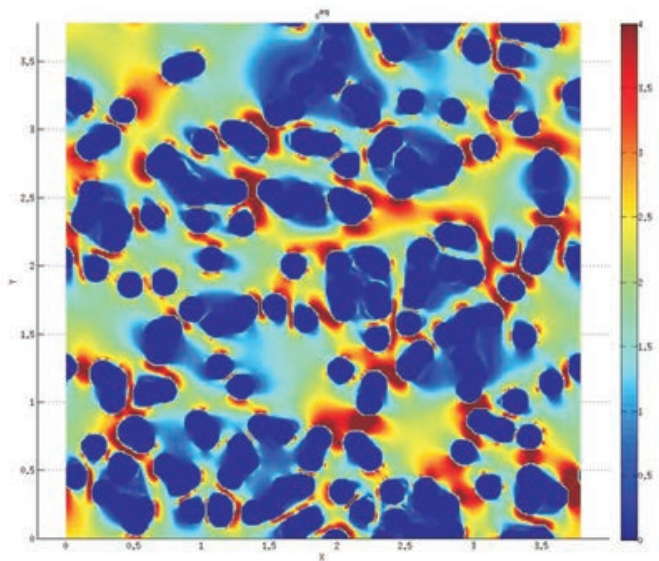

(b)

Figure 25. von Mises stress distribution in the r.v.e. with porous microstructure: (a) SLS and (b) FEM; the maximum stress is threshold to $4 \mathrm{MPa}$.

target volume fraction is $f=0.4$, which leads to an inclusion diameter equal to $D=22.5676$ voxels. As penetration between inclusions is tolerated, the actual volume fraction is lower than the prescribed one and depends on realizations. Pictures of different zooms in the whole domain are shown in figures 26 (a), 27 (a) and 28 (a), corresponding respectively to $5000^{2}$, $1000^{2}$ and $250^{2}$ voxels grids. The macroscopic load is defined as $\bar{\varepsilon}_{11}=1, \bar{\varepsilon}_{22}=1, \bar{\varepsilon}_{12}=0$. We chose $N_{x}^{s}=N_{y}^{s}=80$. The $C_{3}$ criterion was selected to stop iterations. The main objective is to analyze the complexity of the computational times with respect to the number of voxels. Several subdomains with increasing sizes are extracted from the whole domain to carry out the 


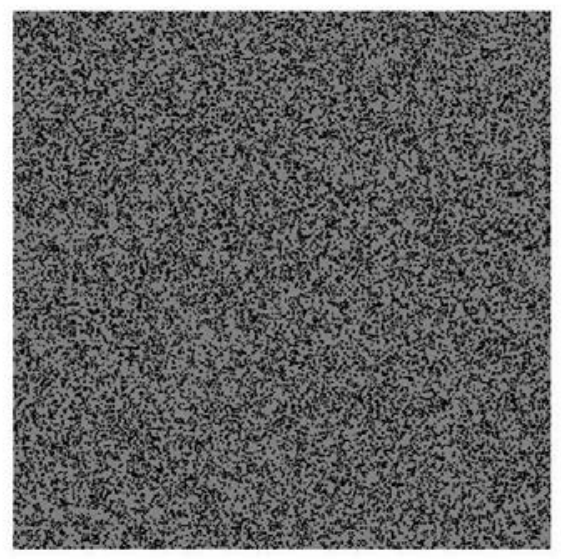

(a)

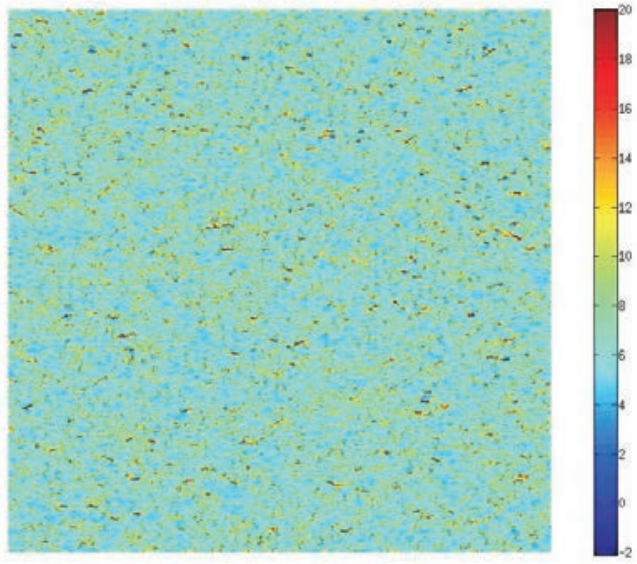

(b)

Figure 26. (a) Random microstructure (1/16) of the whole model, $5000^{2}$ voxel grids; (b) $\sigma_{11}$ stress field.

computations. Stress fields ( $\sigma_{11}$-component) are depicted for the $5000^{2}, 1000^{2}$ and $250^{2}$ voxels grids in figures 26 (b), 27 (b) and 28 (b), respectively. Per-iteration and total computational times are plotted in figure 29, using parallel computations with 32 threads. In this example, the number of iteration depends on the number of voxels, because the problem is different in each case, inclusions being randomly distributed. However, this number stabilizes to 11 and 13 for the largest models. We can note the linear increases of computational times with respect to number of voxels in the domain. The largest problem involves $4.10^{8}$ voxels, corresponding to $8.10^{8}$ degrees of freedom. Such very fine models might be useful for validating future advanced multiscale models. Finally, the evolution of computational times with respect to the number of threads (cores) is plotted in figure 30 , for a $1000 \times 1000$ grid extracted from the whole model.

\subsection{Microstructure obtained from micro tomography}

Finally, we present an example where the phases' geometry is provided from X-ray micro tomography images. The data were kindly provided by prof. Sylvain Meille and Dr. Ing. Jerome Adrien, University of Lyon, CNRS INSA-Lyon, France. As in the present work we only implemented the method in 2D, a single slice of the full $3 \mathrm{~d}$ image is used. We chose $N_{x}^{s}=N_{y}^{s}=80$. The grid contains $500 \times 600$ voxels, as depicted in figure 31. In this figure, the green, blue and brown colors refer to the matrix, inclusions and pores, respectively. The material is a mortar composed of a cement paste (matrix) and sand (inclusions). The material properties of each phases are: $E_{\text {inc }}=3 \mathrm{MPa}, \nu_{\text {inc }}=0.4, E_{\text {mat }}=1 \mathrm{MPa}, \nu_{\text {mat }}=0.1$. The properties of the pores are set to $10^{-6} \mathrm{MPa}$. The properties of the reference medium were chosen as $\lambda^{0}=\mu^{0}=2.5714 \mathrm{MPa}=0.6 \cdot \max (\lambda(\mathbf{x}))$. The macroscopic strain was chosen as $\bar{\varepsilon}_{11}=1, \bar{\varepsilon}_{22}=0, \bar{\varepsilon}_{12}=0$. The computation was run using 32 processors in $105 \mathrm{~s}$ and took 21 iterations using the $C_{3}$ criterion. plots of the von Mises stress fields are depicted in figure 32 and 33 corresponding to direct and modified color scales. 


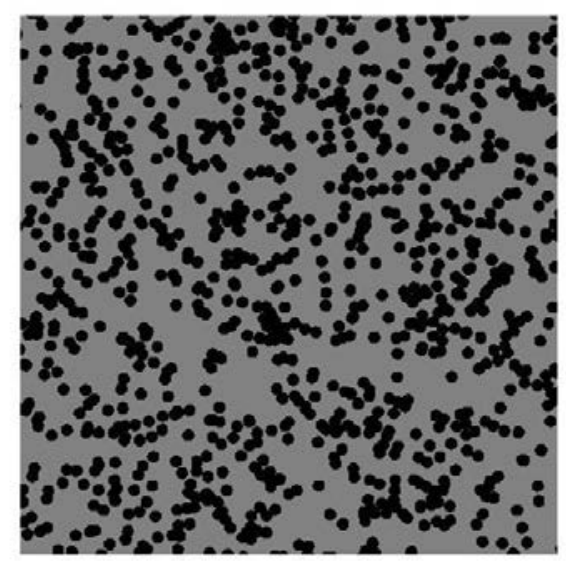

(a)

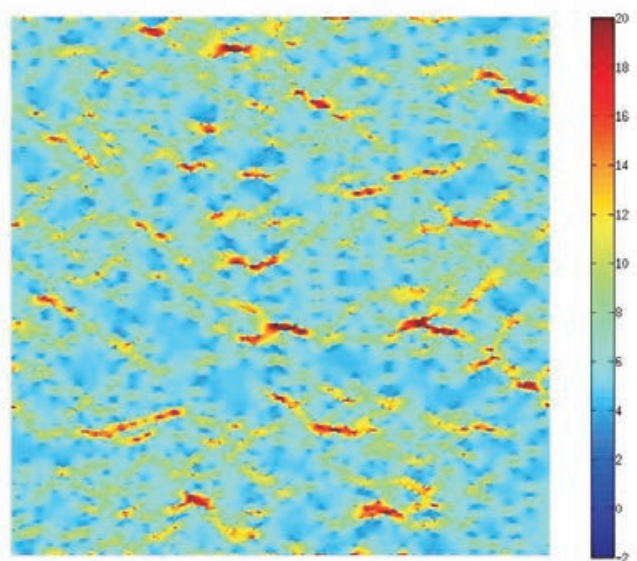

(b)

Figure 27. (a) Random microstructure (1/400) of the whole model, $1000^{2}$ voxel grids; (b) $\sigma_{11}$ stress field.

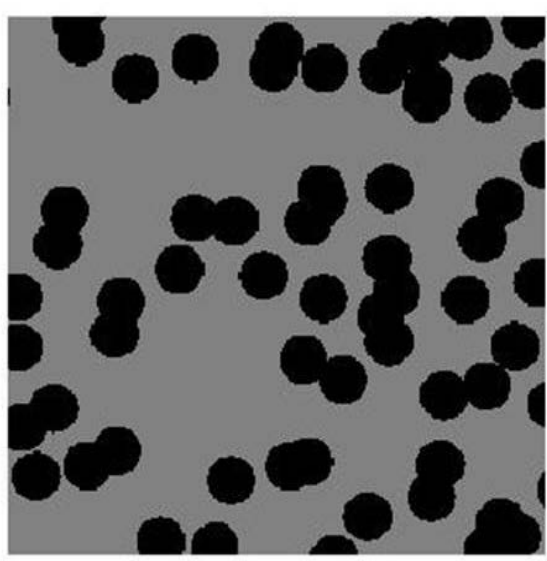

(a)

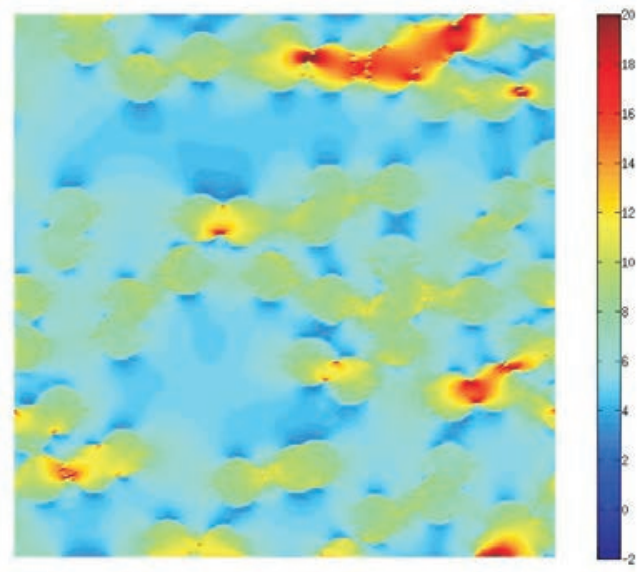

(b)

Figure 28. (a) Random microstructure (1/6400) of the whole model, $250^{2}$ voxel grids; (b) $\sigma_{11}$ stress field.

It is worth noting that data obtained by experimental images of random materials are in general not periodic. If local fields are the aim of the computation, inclusions cut by the boundary will induce artificial singularities in their neighborhood as the resulting periodic phases will involve cut inclusions. This issue might be addressed by extending the model with mirror images of the microstructure. However this process inescapably induces a huge computational effort increase, as the number of voxels must be multiplied by 4 in $2 \mathrm{D}$ and by 8 in 3D. Another possibility is to consider a subdomain which does not contain voxels in the neighborhood of the boundary, to estimate e.g. statistical quantities. If the objective is to 


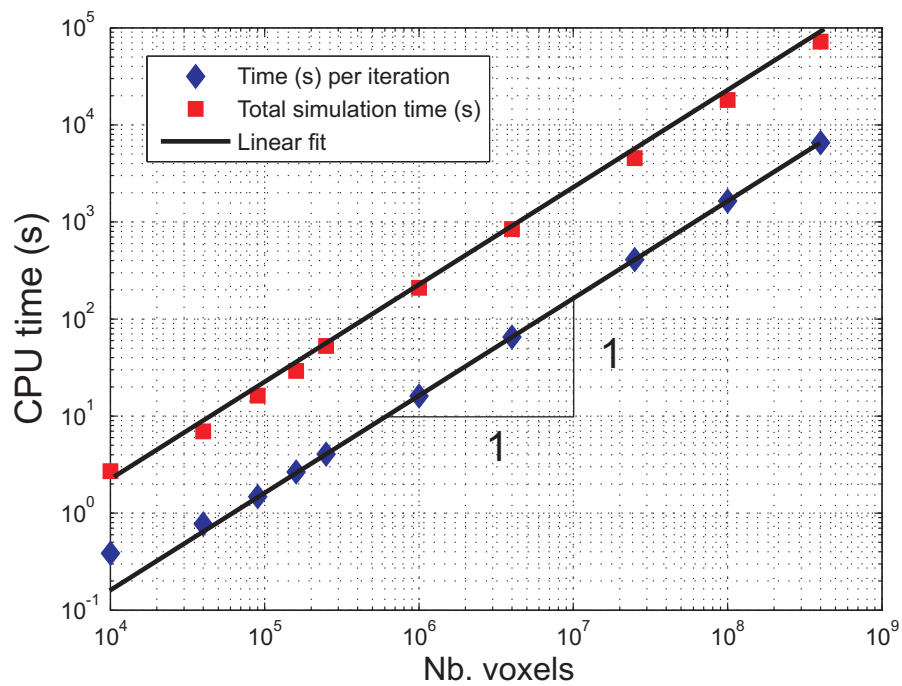

Figure 29. Computational times versus number of voxels.

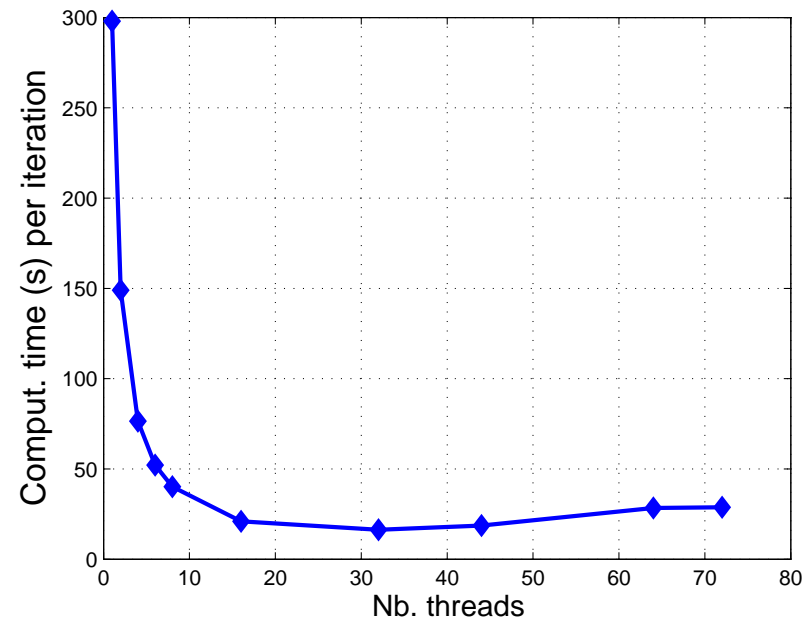

Figure 30. Per-iteration time (s) with respect to the number of threads (cores). 


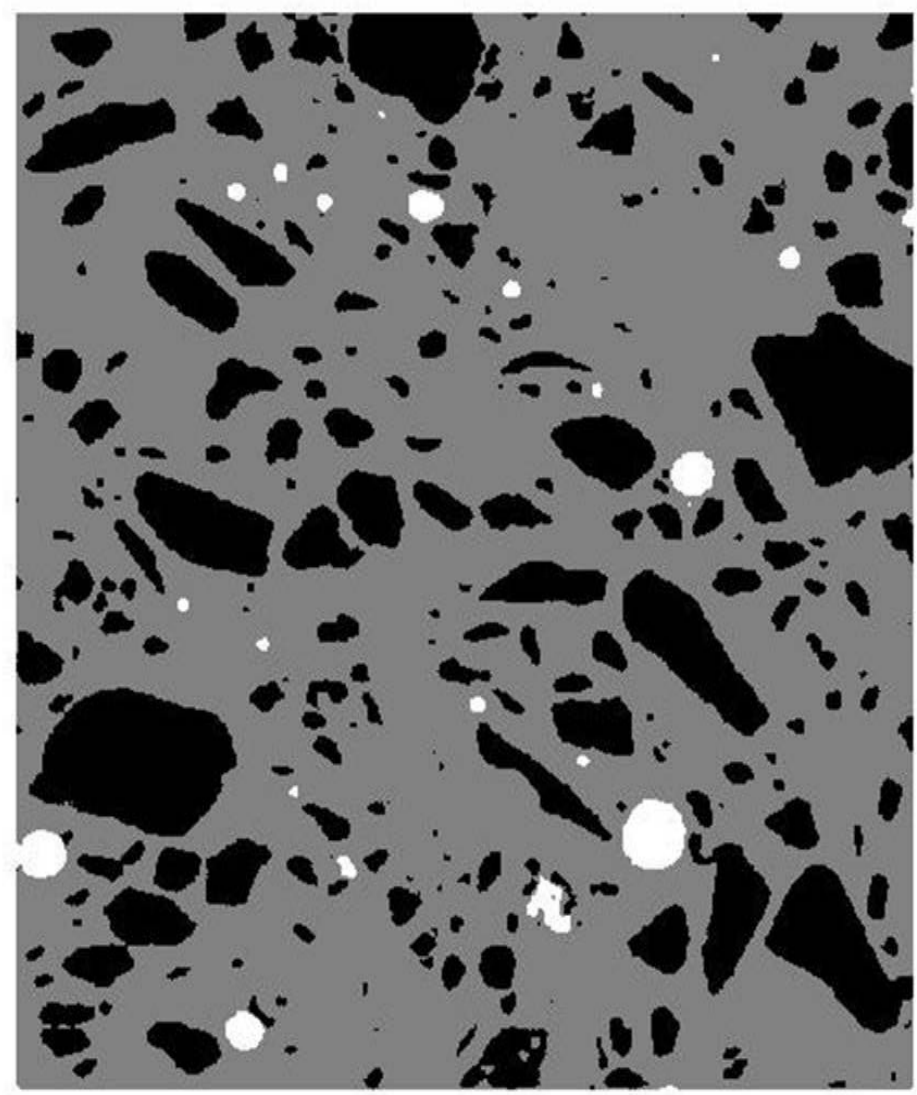

Figure 31. Microtomography image, $500 \times 600$ grid; the gray, black and white phases refer to the matrix, inclusions and pores, respectively.

compute the effective properties, it has been shown (see e.g. [6]) that even if microstructures are non periodic, the periodic boundary conditions lead to the better results regarding convergence of the effective properties with respect to the size of the domain.

\section{Conclusion}

In the proposed work, a new method was proposed, coined as Space Lippmann Schwinger (SLS) technique, avoiding the use of the Fourier transform while maintaining the efficiency and convenience of iterative scheme. In the following approach, no mesh is needed, computations can be carried out in parallel without special modification of the code and the CPU time as well as the memory requirements (which are very low) scale linearly with the number of voxels in the grid. The main ideas have been formulated both in the context of 2D linear steady-state thermal and elastostatics problems. The technique is restricted to periodic microstructures. The different analyses have shown similar dependence to contrast between phases than in 


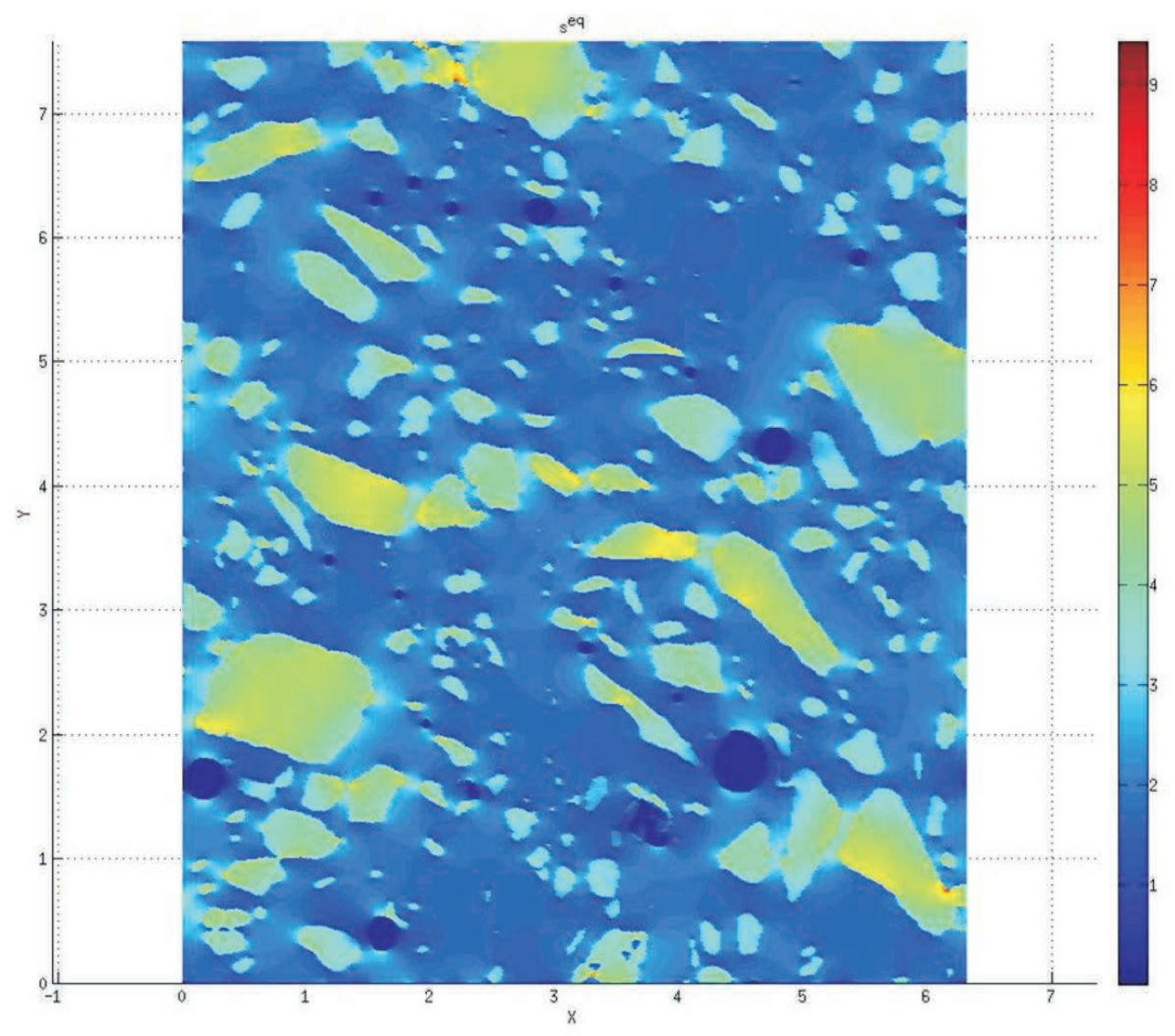

Figure 32. von Mises stress fields computed on the micro tomography image data.

the basic scheme of FFT algorithms. Implementation is very simple, only involving classical FEM computations and loops with sums of matrices. The method is fast and requires low memory, authorizing to solve problems that are not tractable with the finite element method. The method can be extended to nonlinear problems, this will be the aim of a future work.

\section{Aknowledgements}

Fruitful discussions with Professors Guy Bonnet and Vincent Monchiet, University Paris-Est are greatly acknowledged. The author is also grateful to prof. Sylvain Meille and Dr. Ing. Jerome Adrien, University of Lyon, CNRS INSA-Lyon, France, who kindly provided the micro tomography data used for the simulations of this article. 


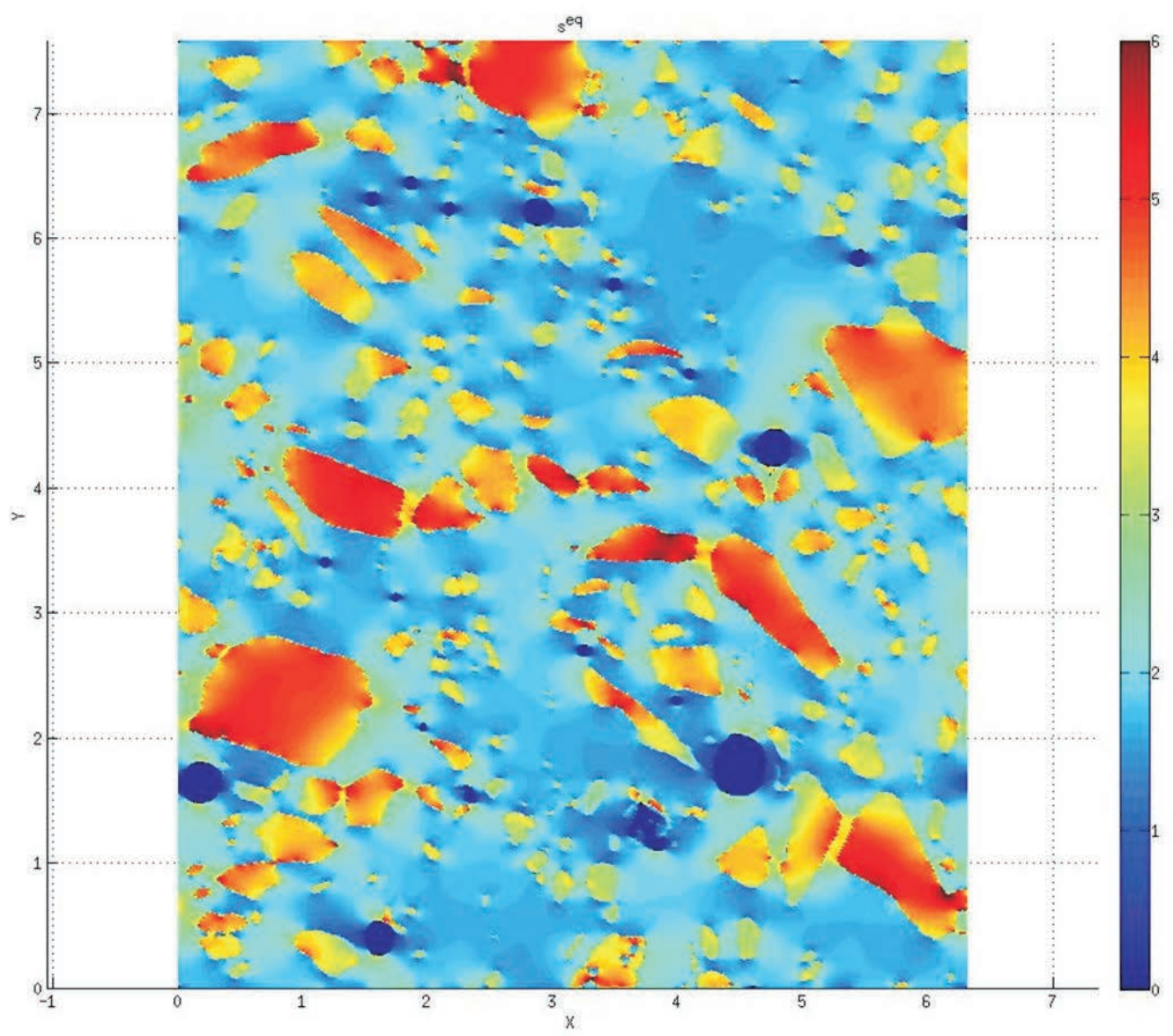

Figure 33. von Mises stress fields computed on the micro tomography image data; the maximum stress is threshold to $6 \mathrm{MPa}$.

7. Appendix: Green functions for thermal and elastostatic problems

\subsection{Thermal steady-state problem}

Let us consider the problem

$$
\nabla \cdot\left(\mathbf{k}^{0} \nabla \mathbf{T}(\mathbf{x})\right)=-r(\mathbf{x}) \text { in } \Omega,
$$

where $r(\mathbf{x})$ is a scalar source term. We can write (62) in index notation as

$$
k_{i j}^{0} T_{, i j}=-r .
$$

Then we have

$$
T(\mathbf{x})=-\int_{\Omega} G(\mathbf{x}-\mathbf{y}) r(\mathbf{y}) d \Omega,
$$

Copyright (c) 2011 John Wiley \& Sons, Ltd. Prepared using nmeauth.cls

Int. J. Numer. Meth. Engng 2011; 00:1-1 
where $G(\mathbf{x}, \mathbf{y})$ is the Green function such that

$$
k_{i j}^{0} G_{, i j}(\mathbf{x}-\mathbf{y})=-\delta(\mathbf{x}-\mathbf{y}) .
$$

where $\delta(\mathbf{x}-\mathbf{y})$ is the Dirac's delta function. It yields

$$
\nabla T_{i}(\mathbf{x})=\int_{\Omega} G_{, i}(\mathbf{x}-\mathbf{y}) r(\mathbf{y}) d \Omega .
$$

An explicit expression of the Green function in the case of isotropic conduction can be found e.g. in [9]. Now let us consider the case where we seek at a direct linear relationship between the temperature solution and the components of a vector flux field $\mathbf{q}(\mathbf{x})$ such as

$$
\nabla \cdot\left(\mathbf{k}^{0} \nabla \mathbf{T}(\mathbf{x})\right)=-\nabla \cdot(\mathbf{q}(\mathbf{x})) \text { in } \Omega,
$$

arising for example from Eq. (9). In index notation we obtain

$$
k_{i j}^{0} T_{, i j}=-q_{i, i} .
$$

Then the solution can be expressed as

$$
T(\mathbf{x})=-\int_{\Omega} G_{i}(\mathbf{x}-\mathbf{y}) q_{i}(\mathbf{y}) d \Omega
$$

where $G(\mathbf{x}, \mathbf{y})$ is such that

$$
k_{i j}^{0} G_{m, i j}(\mathbf{x}-\mathbf{y})=-\delta_{, i}(\mathbf{x}-\mathbf{y}) \delta_{i m}
$$

where $\delta_{i j}=1$ if $i=j$ and zero otherwise, and

$$
\nabla T_{i}(\mathbf{x})=\int_{\Omega} G_{i, j}(\mathbf{x}-\mathbf{y}) q_{j}(\mathbf{y}) d \Omega=\int_{\Omega} \Gamma_{i j}^{0}(\mathbf{x}-\mathbf{y}) q_{j}(\mathbf{y}) d \Omega .
$$

\subsection{Linear elasticity}

Let us consider the equilibrium equation for small strain elasticity:

$$
\nabla \cdot\left(\mathbb{C}^{0}: \varepsilon(\mathbf{x})\right)=-f(\mathbf{x}) \text { in } \Omega,
$$

where $f$ is a vector body force. Eq. (72) can be rewritten as

$$
C_{i j k l}^{0} u_{k, l j}=-f_{i} \text {. }
$$

The displacement solution can be expressed by means of a Green function as

$$
u_{i}(\mathbf{x})=\int_{\Omega} G_{i j}(\mathbf{x}-\mathbf{y}) f_{j}(\mathbf{y}) d \Omega
$$

where $G_{i j}(\mathbf{x}, \mathbf{y})$ is such that

$$
C_{i j k l} G_{k m, l j}=-\delta(\mathbf{x}-\mathbf{y}) \delta_{i m} .
$$


Then

$$
\varepsilon_{i j}(\mathbf{x})=\int_{\Omega} G_{i k, j}(\mathbf{x}-\mathbf{y}) f_{k}(\mathbf{y}) d \Omega .
$$

An explicit expression of the elastic isotropic Green function in 2D and 3D can be found e.g. in [9], [3]. In the case where we seek a linear relation between the displacements and the effects of a symmetric, second-order eigenstress tensor field $\boldsymbol{\tau}(\mathbf{x})$, arising for example from Eq. (30), we have

$$
\nabla \cdot\left(\mathbb{C}^{0}: \varepsilon(\mathbf{x})\right)=-\nabla \cdot(\boldsymbol{\tau}(\mathbf{x})) \text { in } \Omega,
$$

or

$$
C_{i j k l} u_{k, l j}=-\tau_{i j, j} .
$$

Then the displacements field is expressed by

$$
u_{i}(\mathbf{x})=\int_{\Omega} G_{i j k}(\mathbf{x}-\mathbf{y}) \tau_{j k}(\mathbf{y}) d \Omega
$$

where $G_{i j k}(\mathbf{x}, \mathbf{y})$ is such that

$$
C_{i j k l} G_{k m p, l j}=-J_{i j}^{m p} \delta_{, j}(\mathbf{x}-\mathbf{y})
$$

and where the second-order tensor $\mathbf{J}^{m p}$ is defined by

$$
\mathbf{J}^{m p}=\frac{2-\delta_{m p}}{2}\left(\mathbf{e}_{m} \otimes \mathbf{e}_{p}+\mathbf{e}_{p} \otimes \mathbf{e}_{m}\right) .
$$

The form of $\mathbf{J}^{m p}$ is due to the fact that that $\boldsymbol{\tau}$ is assumed symmetric. Then we obtain:

$$
\varepsilon_{i j}(\mathbf{x})=\int_{\Omega} G_{i k l, j}(\mathbf{x}-\mathbf{y}) \tau_{k l}(\mathbf{y}) d \Omega=\int_{\Omega} \Gamma_{i j k l}^{0}(\mathbf{x}-\mathbf{y}) \tau_{k l}(\mathbf{y}) d \Omega .
$$

\section{REFERENCES}

1. G. Bonnet (2007). Effective properties of elastic periodic composites media with fibers. Journal of the Mechanics and Physics of Solids, 40:3647-3679

2. S. Brisard, L. Dormieux (2010). FFT-based methods for the mechanics of composites: A general variational framework. Computational Materials Science, 49(3):663-671

3. V.A. Buryachenko, Micromechanics of Heterogeneous Materials. Springer, New-York, 2007

4. S. Li, G. Wang, Introduction to micromechanics and nanomechanics. World Scientific Publishing, 2008

5. Q.-C. He, Q.-S. Zheng (1996). On the symmetry of $2 D$ elastic and hyperelastic tensors. Journal of Elasticity, $43: 203-225$

6. T. Kanit, S. Forest, J. Galliet, V. Mounoury, D. Jeulin (2003). Determination of the siez of the representative volume element for random composites: statistical and numerical approach. International Journal of Solids ans Structures, 40:3647-3679

7. R. Kress, Linear integral equations. Springer Verlag, 1999

8. E. Kroner, Statistical continuum mechanics. Springer Verlag, Wien, 1972

9. S. Li, G. Wang, Introduction to micromechanics and nanomechanics. World Scientific Publishing, 2008

Int. J. Numer. Meth. Engng 2011; 00:1-1 
10. J.C. Michel, H. Moulinec, P. Suquet (1999). Effective properties of composite materials with periodic microstructure: a computational approach. Computer Methods in Applied Mechanics and Engineering, 172:109-143

11. J.C. Michel, H. Moulinec, P. Suquet (2000). A computational method based on augmented Lagrangians and Fast Fourier transforms for composites with high contrast. Computer Modelling in Engineering and Sciences, 1(2):79-88

12. G.W. Milton, The theory of Composites. Cambridge University Press, 2002.

13. J.C. Michel, H. Moulinec, P. Suquet (2001). A computational scheme for linear and nonlinear composites with arbitrary phase contrast. International Journal for Numerical Methods in Engineering, 52:139-169

14. V. Monchiet, G. Bonnet, G. Lauriat (2009). A FFT-based method to compute the permeability induced by a Stokes slip flow through a porous medium. Comptes Rendus de Mécanique, 337(4):192-197

15. V. Monchiet, G. Bonnet (2011). A polarization-based FFT iterative scheme for computing the effective properties of elastic composites with arbitrary contrast. International Journal for Numerical Methods in Engineering, in press, DOI: 10.1002/nme.3295

16. H. Moulinec, P. Suquet (1994). A fast numerical method for computing the linear and nonlinear properties of composites. Comptes Rendus de l'Académie des Sciences Paris II, 318:1417-1423

17. H. Moulinec, P. Suquet, (1998). A numerical method for computing the overall response of nonlinear composites with complex microstructures. Computer Methods in Applied Mechanics and Engineering, 157:69-94

18. H. Moulinec, P. Suquet, (2003). Comparison of FFT-based methods for computing the response of composites with highly contrasted mechanical properties. Physica B, 338:58-60

19. W.H. Müller, (1996). Mathematical versus experimental stress analysis of inhomogeneities in solids. Journal de Physique IV, 6:C1.139-C1.148

20. L. Salvo, P. Cloetens, E. Maire, S. Zabler, J.J. Blandin, J.Y. Buffière, W. Ludwig, E. Boller, D. Bellet, C. Josserond (2003). X-ray micro-tomography an attractive characterisation technique in materials science. Nuclear Instruments and Methods in Physics Research B, 200:273-286

21. V. Šmilauer, Z.P. Bažant (2010). Identification of viscoelastic $C-S-H$ behavior in mature cement paste by FFT-based homogenization method. Cement and Concrete Research, 40:197-207

22. P. Spanne, J.F. Thovert, C.F. Jacquin, W.B. Lindquist, K.W. Jones, P.M. Adler (1999). Synchrotron commputed microtomography of porous media - topology and transports. Physical Review Letters, 73(14):2001-2004

23. S. Torquato (1997). Effective stiffness tensor of composite media-I. Exact series expansion. Journal of the Mechanics and Physics of Solids, 45:1421-1448

24. V. Vinagradov, G.W. Milton (2008). An accelerated FFT algorithm for thermoelastic and nonl-linear composites. International Journal for Numerical Methods in Engineering, 76:1678-1695

25. F. Willot, Y-P. Pellegrini, M.J. Idiart, P. Ponte Castañeda, (2009). Effective medium theory for infinitecontrast two-dimensionally periodic linear composites with strongly anisotropic matrix behavior: Dilute limit and crossover behavior. Physical review B, 78(1):1-17

26. F. Willot, D. Jeulin, (2009). Elastic behavior of composites containing Boolean random sets of inhomogeneities. International Journal of Engineering Science, 47:313-324

27. J. Zeman, J. Vondrejc, J. Novác, I. Marek, (2010). Accelerating a FFT-based solver for numerical homogenization of periodic media by conjugate gradients. Journal of Computational Physics, 229:80658071 